ARTICLE

Received 20 Apr 2014 | Accepted 1 Jul 2014 | Published 31 Jul 2014 DOl: 10.1038/ncomms5564

\title{
Enhancing the fidelity of neurotransmission by activity-dependent facilitation of presynaptic potassium currents
}

Yi-Mei Yang ${ }^{1,2, \star}$, Wei Wang ${ }^{3,4, \star}$, Michael J. Fedchyshynn ${ }^{1,2}$, Zhuan Zhou ${ }^{5}$, Jiuping Ding ${ }^{3}$ \& Lu-Yang Wang ${ }^{1,2}$

Neurons convey information in bursts of spikes across chemical synapses where the fidelity of information transfer critically depends on synaptic input-output relationship. With a limited number of synaptic vesicles (SVs) in the readily releasable pool (RRP), how nerve terminals sustain transmitter release during intense activity remains poorly understood. Here we report that presynaptic $\mathrm{K}^{+}$currents evoked by spikes facilitate in a $\mathrm{Ca}^{2+}$-independent but frequency- and voltage-dependent manner. Experimental evidence and computer simulations demonstrate that this facilitation originates from dynamic transition of intermediate gating states of voltage-gated $\mathrm{K}^{+}$channels (Kvs), and specifically attenuates spike amplitude and inter-spike potential during high-frequency firing. Single or paired recordings from a mammalian central synapse further reveal that facilitation of Kvs constrains presynaptic $\mathrm{Ca}^{2+}$ influx, thereby efficiently allocating SVs in the RRP to drive postsynaptic spiking at high rates. We conclude that presynaptic Kv facilitation imparts neurons with a powerful control of transmitter release to dynamically support high-fidelity neurotransmission.

\footnotetext{
${ }^{1}$ Program in Neurosciences and Mental Health, SickKids Research Institute, Toronto, Ontario, Canada M5G 1X8. ${ }^{2}$ Department of Physiology, University of Toronto, Toronto, Ontario, Canada M5S 1A8. ${ }^{3}$ Key Laboratory of Molecular Biophysics of the Ministry of Education, Huazhong University of Science and Technology, Wuhan 430074, China. ${ }^{4}$ Department of Medical Engineering, the 180th Hospital of PLA, Quanzhou 362000, China. ${ }^{5}$ Institute of Molecular Medicine \& PKU-IDG/McGovern Institute for Brain Research, Peking University, Beijing 100871, China. * These authors contributed equally to this work. Correspondence and requests for materials should be addressed to J.D. (email: jpding@mail.hust.edu.cn) or to L-Y.W. (email: luyang.wang@utoronto.ca).
} 
$\mathrm{C}$ lassical work by Hodgkin and Huxley ${ }^{1}$ ingeniously described single action potentials (APs or spikes) in the squid giant axon with mathematical expressions of intricate time- and voltage-dependent changes in two conductances: $\mathrm{Na}^{+}$and $\mathrm{K}^{+}$conductance. However, overwhelming evidence suggests that central neurons integrate a large number of ion channels to generate single APs and more complex patterns of spikes to encode information ${ }^{2-11}$. Among these channels, $\mathrm{K}^{+}$channels are the most diverse of all, containing 10 subfamilies with a total number of subunits exceeding 70 (ref. 12). Different combinations of subunits from the same and/or different subfamily of $\mathrm{K}^{+}$channels give rise to tremendous heterogeneity in neuronal excitability, spike waveform and firing patterns manifested by distinct populations of neurons in the mammalian brain. Aside from the diversity of native $\mathrm{K}^{+}$ channels, functional and structural studies have further extended the simple gating states that Hodgkin and Huxley ${ }^{1}$ originally implicated (that is, $0-4$ gating particles) to show that each channel gates in much more complicate schemes. Indeed, computational modelling of $\mathrm{K}^{+}$and other ion channels suggest that highly dynamic transition between multiple gating states, including several closed states, open state and in some cases inactivation states, takes place to account for macroscopic behaviour of ion channels that underlies various forms of firing activity typically recoded from soma or dendrites of neurons ${ }^{3-5,8-11,13}$. However, very little is known about whether and how the intermediate gating states of voltage-gated channels contribute to the presynaptic excitability and transmitter release in a physiological context.

To this end, we investigate the properties of voltage-gated $\mathrm{K}^{+}$ channels (Kvs) at the calyx of Held nerve terminal in the mouse brainstem and several other fast-spiking neurons in hippocampus and cerebellum with short bursts of native or pseudo-APs. We find that currents mediated by Kvs facilitate in a frequencydependent but $\mathrm{Ca}^{2+}$-independent fashion. With further experiments and computer simulations using the Markov kinetic modelling of ion channels at the presynaptic calyces ${ }^{14-16}$, we reveal that this facilitation resides in the intermediate closed states of Kvs, independent of any diffusible second messengers, and affects the synaptic input-output relationship. Our observations suggest that the enhancement of Kvs fine-tunes presynaptic spikes, and critically regulates the magnitude and polarity of short-term synaptic plasticity to dynamically promote highfidelity neurotransmission.

\section{Results}

Neuronal $\mathrm{K}^{+}$currents facilitate during repetitive activity. In the mammalian brain, fast-spiking neurons fire regularly at several $100-1,000 \mathrm{~Hz}$ with little adaptation, and have crucial roles in gating input detection, integration and information coding. For example, cerebellar Purkinje cells (PCs) and principal neurons in the auditory brainstem rapidly transmit motor and sensory signals with temporal precision, while inhibitory interneurons in the cortical or subcortical areas project strong inhibition to modulate the local network activity important for a variety of cognitive functions. To directly investigate biophysical basis of the fastspiking modality, we first isolated and recorded $\mathrm{K}^{+}$currents $\left(I_{\mathrm{K}}\right)$ from several fast-spiking central neurons, including hippocampal dentate gyrus interneurons (DGIs), cerebellar stellate neurons (SNs) and PCs, as well as the calyx of Held synapse in the auditory brainstem, under the condition that $\mathrm{Na}^{+}$and $\mathrm{Ca}^{2+}$ channels were blocked by extracellular tetrodotoxin (TTX; 0.5$1 \mu \mathrm{M})$ and cadmium chloride $\left(\mathrm{CdCl}_{2} ; 20 \mu \mathrm{M}\right)$, respectively. We evoked $I_{\mathrm{K}}$ by a pair of identical pseudo-APs at incremental intervals with their waveforms mimicking native spikes at room $\left(22^{\circ} \mathrm{C}\right)$ or near-physiological temperature $\left(35^{\circ} \mathrm{C}\right)$ from these neurons (Fig. 1 and Supplementary Fig. 1). Unexpectedly, we found that $I_{\mathrm{K}}$ activated by the second spikes significantly enhanced in all cases. Figure 1a,b illustrated from the calyx of Held nerve terminal, the amplitude and area integral of $I_{\mathrm{K}}$ evoked by the second spike facilitated by $142.4 \pm 8.3 \%$ (mean \pm s.e.m.) and $153.3 \pm 7.5 \%$ relative to that by the first spike at the interval of $1 \mathrm{~ms}$, respectively (AP waveform: -80 to $+30 \mathrm{mV}$, rise time: $0.2 \mathrm{~ms}$, decay time: $0.4 \mathrm{~ms}$ ). This facilitation decreased as the interval increased and diminished completely within $10 \mathrm{~ms}$. The time-dependent decline in paired-pulse facilitation (PPF) by measuring the amplitude or area integral of $I_{\mathrm{K}}$ can be well described by single exponential functions with similar time constants $(\tau)$ of 0.6 or $0.7 \mathrm{~ms}$ (Fig. 1b). This phenotype was also evident for $I_{\mathrm{K}}$ recorded from hippocampal DGIs, cerebellar SNs and PCs (Supplementary Fig. 1a-c). To determine whether PPF of Kvs is restricted to fast-spiking cells, we investigated $I_{\mathrm{K}}$ from hippocampal CA1 neurons (CA1s), which are well known for firing at low frequencies. Similar to the fast-spiking neurons, $I_{\mathrm{K}}$ in CA1s evoked by a pair of pseudo-APs facilitated and the PPF reduced exponentially over prolonged intervals as well (Supplementary Fig. 2). These observations suggest that Kvs in many types of neurons facilitate, although the extent and decay time course of PPF are different, likely depending on the composition of native $\mathrm{K}^{+}$channels and experimental conditions.

Given the intriguing feature of $I_{\mathrm{K}}$ evoked by closely spaced APs, we reasoned that $\mathrm{Kv}$ facilitation $(\mathrm{KvF})$ might be important for neurons to project their outputs onto the target cells. Therefore, we subsequently focused on the calyx of Held synapse, which is an axosomatic synapse capable of faithfully transmitting at ultra-fast speed (up to $1 \mathrm{kHz}$ ) to reliably convey interaural timing and intensity cues for sound localization in the auditory brainstem ${ }^{17-20}$. Unlike tiny presynaptic en passant boutons from the axons of other fast-spiking neurons, the large calyceal terminals are readily accessible with patch-clamp electrodes and can serve as an ideal model to study the mechanisms for $\mathrm{KvF}$ and its impact on presynaptic spikes and downstream synaptic signalling. Previous work has demonstrated that there are two major subtypes of Kvs at the calyces, namely low-threshold $\alpha$ dendrotoxin (DTX)-sensitive Kv1s (K-LT) and high-threshold tetraethylammonium (TEA)-sensitive Kv3s (K-HT) that activate at -40 and $-20 \mathrm{mV}$, and regulate the spike amplitude and width, respectively $18,19,21-23$. To directly test whether the two subtypes of Kvs with distinct kinetics and voltage dependence contribute to the facilitation differently, we performed paired-pulse experiments in the presence of either DTX or TEA to isolate currents mediated by K-HT $\left(I_{\mathrm{K}-\mathrm{HT}}\right)$ or K-LT $\left(I_{\mathrm{K}-\mathrm{LT}}\right)$ in the outside-out patch configuration. This recording mode eliminates potential contamination from other $\mathrm{K}^{+}$channels in distal axons and space-clamp errors associated with whole-cell recordings from morphologically complex calyces. We found both $I_{\mathrm{K}-\mathrm{HT}}$ and $I_{\mathrm{K}-\mathrm{LT}}$ exhibited prominent PPF but the profile of their PPF was clearly distinct (Fig. 1c,d). The PPF for $I_{\mathrm{K}-\mathrm{HT}}$ $(179.6 \pm 10.2 \%$ at $1 \mathrm{~ms})$ was much less than that for $I_{\mathrm{K}-\mathrm{LT}}$ $(302.8 \pm 32.9 \%$ at $1 \mathrm{~ms})$ and declined markedly faster $(\tau=0.3 \mathrm{~ms}$ for $I_{\mathrm{K}-\mathrm{HT}}$ versus $\tau=0.6 \mathrm{~ms}$ for $I_{\mathrm{K}-\mathrm{LT}}$; Fig. 1e,f). These results demonstrate that activity-dependent facilitation is present among many subtypes of Kvs, and differences in the characteristics of their PPF are likely because of heterogeneous gating kinetics of each subtype of Kvs.

Interestingly, when brief voltage steps were introduced to the outside-out patches in between a pair of pseudo-APs at the interval of $1.2 \mathrm{~ms}$ where facilitation of $I_{\mathrm{K}}$ was robust at $-80 \mathrm{mV}$ $(168.8 \pm 4.4 \%)$, we found that PPF was attenuated by hyperpolarizing steps and eliminated $(\mathrm{PPF}=107.4 \pm 3.3 \%)$ at the nonphysiologically low potential of $-140 \mathrm{mV}$. Conversely, a small 
sub-threshold depolarization $(10 \mathrm{mV})$ further enhanced facilitation of $I_{\mathrm{K}}$ to $185.6 \pm 15.2 \%$ at $-70 \mathrm{mV}$ (Fig. 2a), indicating a priming effect of small depolarization on the magnitude of PPF. When the amplitude of pseudo-APs was increased from 100 to $150 \mathrm{mV}$ while the rise and decay time remained unchanged, we found that the $I_{\mathrm{K}}$ increased accordingly, however, the extent of PPF linearly decreased (Fig. 2b,c). Collectively, our experiments suggest that $\mathrm{KvF}$ is highly voltage dependent and inversely correlates with the inter-spike interval and spike amplitude, implying that previously activated Kvs may have a strong impact on the behaviour of Kvs in response to subsequent stimuli.

$\mathrm{KvF}$ is frequency dependent but $\mathrm{Ca}^{2+}$ independent. To examine whether $\mathrm{KvF}$ occurs during physiological activity, we recorded $I_{\mathrm{K}}$ from the mature calyceal terminals using previously obtained native AP trains at various frequencies $(200-400 \mathrm{~Hz})$ as voltage-clamp templates (Fig. 3 and Supplementary Fig. 3). Typically, these spikes displayed a slight use-dependent reduction in amplitude and persistent plateau potential resulting from depolarizing after potential (DAP) driven by resurgent $\mathrm{Na}^{+}$ currents $\left(I_{\mathrm{Na}}\right)^{24}$. Figure $3 \mathrm{a}$ showed an example of presynaptic $I_{\mathrm{K}}$ (with $\mathrm{P} / 4$ digital subtraction of leak and capacitive currents) evoked by $400 \mathrm{~Hz}$ AP trains, where the $I_{\mathrm{K}}$ amplitude increased significantly in the first 5-10 spikes and sustained throughout the entire train at a level of $20-30 \%$ above the first response (first $I_{\mathrm{K}}$ : $1.35 \pm 0.21 \mathrm{nA}, 10$ th $I_{\mathrm{K}}: 1.65 \pm 0.25 \mathrm{nA}$; Fig. 3c). Such facilitation was readily reversed upon cessation of stimulation, and the magnitude of facilitation depended on the AP frequency and DAP (Supplementary Fig. 3). The profile of $I_{\mathrm{K}}$ facilitation was very similar to that estimated by pharmacologically isolated (that is, TEA and DTX sensitive) currents without $\mathrm{P} / \mathrm{N}$ leak subtraction, indicating that this feature of Kvs cannot be attributed to the voltage-clamp errors (Supplementary Fig. 4). Similar characteristics of $I_{\mathrm{K}}$ were observed from hippocampal DGIs, cerebellar SNs and PCs at 22 or $35^{\circ} \mathrm{C}$ (Supplementary Fig. 1d-f). A prominent example was $\mathrm{SNs}$ where realistic AP trains at $400 \mathrm{~Hz}$ generated $>300 \%$ facilitation of $I_{\mathrm{K}}$ at physiological temperature either from the whole cells or outside-out patches. In contrast, $I_{\mathrm{K}}$ evoked by native APs at $50 \mathrm{~Hz}$ in slow-spiking CA1 neurons markedly decreased, likely as a result of use-dependent inactivation of the Kvs (Supplementary Fig. 2). These results demonstrate that $\mathrm{KvF}$ during natural bursts of activity is frequency dependent, being most noticeable in fast neurons at high-frequency firing but largely absent in slowspiking neurons.

Because APs not only activate Kvs but also voltage-gated $\mathrm{Ca}^{2+}$ channels (VGCCs), which may allow $\mathrm{Ca}^{2+}$ influx into the a Paired pulses (AP-like voltage command)

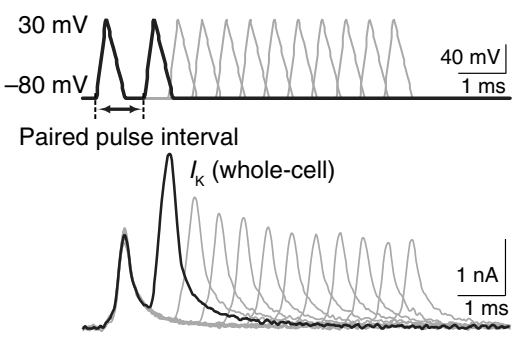

b

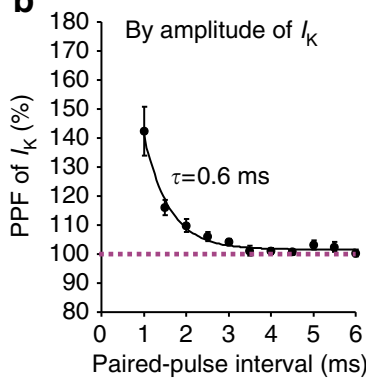

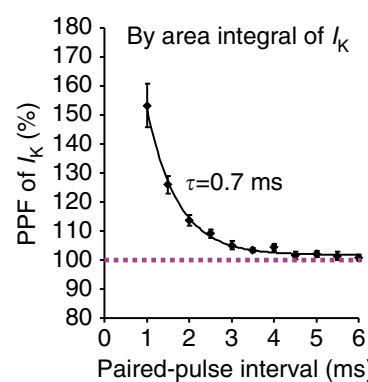
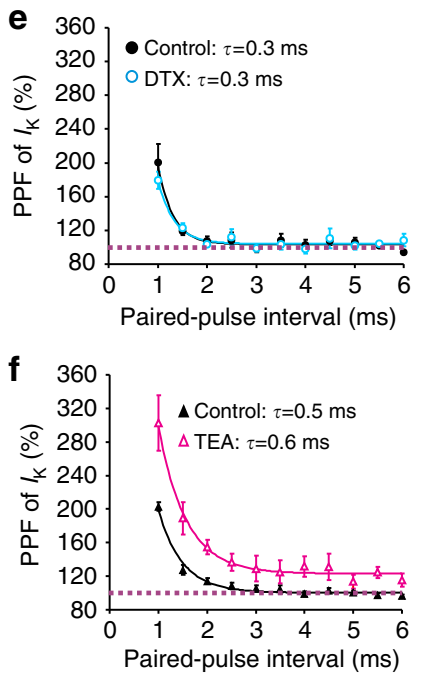
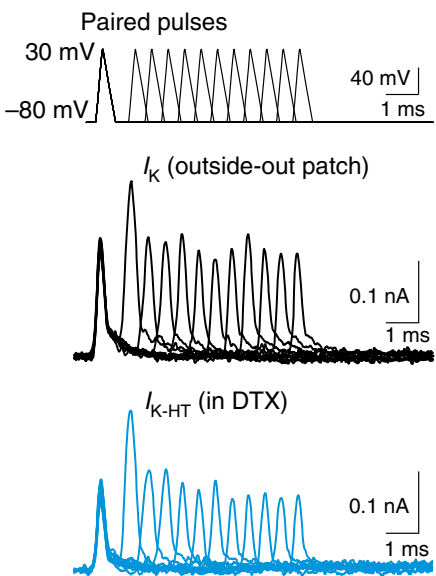

d
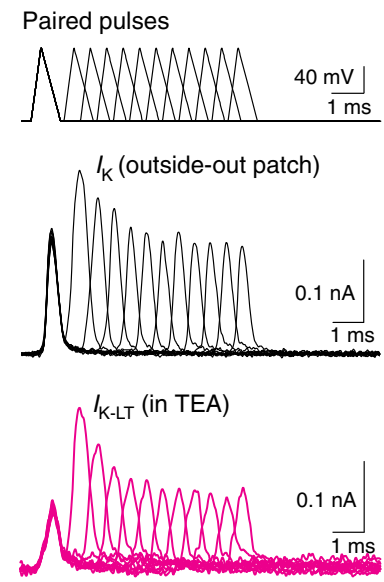

Figure 1 | Paired-pulse facilitation (PPF) of native $\mathbf{K}^{+}$currents from the central nerve terminal. (a) Potassium currents $\left(I_{K}\right.$, bottom panel) obtained from the calyx of Held nerve terminal in the whole-cell mode in response to a pair of action potential (AP)-like voltage command with variable intervals from 1 to $6 \mathrm{~ms}$ (AP waveform: -80 to $+30 \mathrm{mV}$, rise time: $0.2 \mathrm{~ms}$, decay time: $0.4 \mathrm{~ms}$, top panel). The inter-pulse interval is illustrated as the time difference between the starting points of two pulses. (b) PPF, defined as the ratio of the amplitude (left) or area integral (right) of the $2 \mathrm{nd} l_{\mathrm{K}}$ versus that of the $1 \mathrm{st}$ responses, declines over increasing time intervals. The decay rates of PPF are fit with a single exponential function and the time constants $(n=9)$ are given in this and following figures. (c,d) Representative traces of $I_{K}$ recorded from outside-out patches of the nerve terminal in the absence (black traces, middle panels) or presence of $\alpha$-dendrotoxin (DTX, blue traces, $\mathbf{c}$ ) or tetraethylammonium (TEA, magenta traces, $\mathbf{d}$ ) in response to the paired-pulse paradigms (top panels: amplitude: -80 to $+30 \mathrm{mV}$; half-width: $0.3 \mathrm{~ms}$ in $\mathbf{c}, 0.45 \mathrm{~ms}$ in $\mathbf{d}$ ). (e,f) Summaries of the extent of PPF (quantified by area integral of $I_{K}$ ) with or without DTX $(n=6)$ or TEA $(n=7)$ over inter-pulse intervals. Error bars indicate \pm s.e.m. 1st, first; 2 nd, second. 

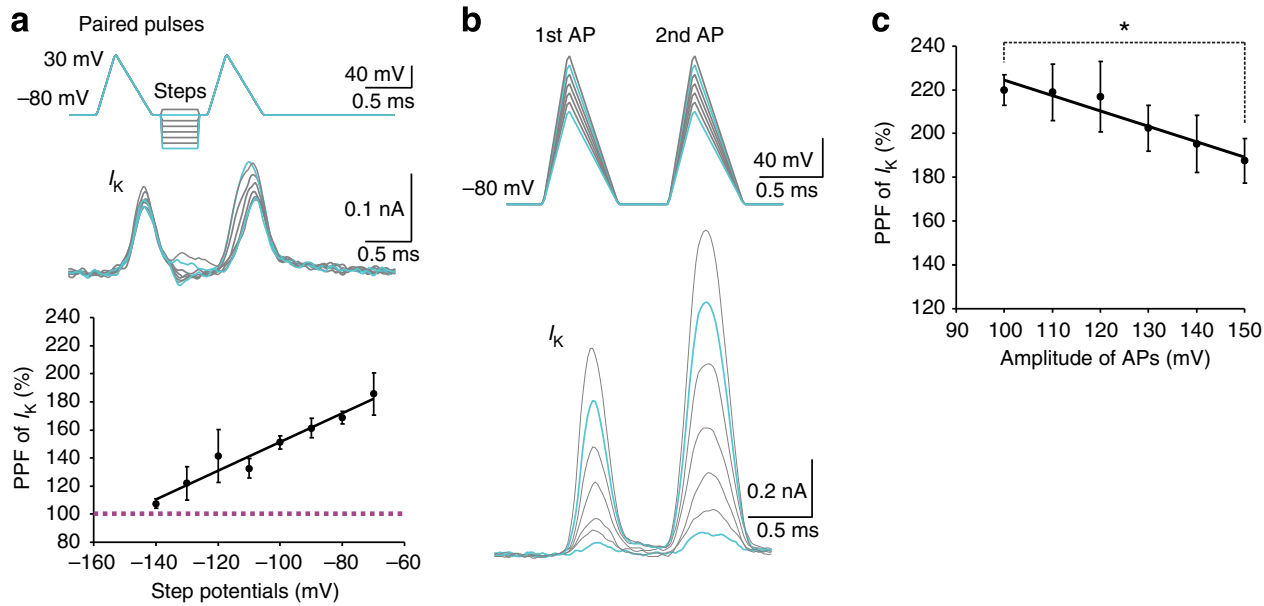

Amplitude of APs (mV)

Figure 2 | PPF of $I_{\mathbf{K}}$ depends on the voltage and amplitude of AP-like pulses. (a) $I_{K}$ (middle panel) from outside-out patches triggered by paired pulses that are separated by a brief voltage step $(0.4 \mathrm{~ms}$ ) ranging from -140 to $-70 \mathrm{mV}$ (top panel). Note that the hyperpolarization step of $-140 \mathrm{mV}$ eliminates facilitation of $I_{K}$ in contrast to the step of $-80 \mathrm{mV}$ (turquoise). Average PPF at different potentials is summarized in the bottom panel ( $n=5$ ). (b) Paired pulses (top) with increasing amplitude from 100 to $150 \mathrm{mV}$ used to evoke $I_{\mathrm{K}}$ (bottom) from outside-out patches. $I_{\mathrm{K}}$ produced by lower (100 mV) or higher $(150 \mathrm{mV})$ paired pulses are compared (turquoise). (c) The decline of PPF over the pulse amplitude is fitted with a linear regression $(n=6)$. The asterisk indicates statistical significance $(P=0.009$, two-tailed paired Student's $t$-tests) between PPF evoked by the lower and that by the higher pulses. Error bars denote \pm s.e.m.

nerve terminal to either open $\mathrm{Ca}^{2+}$-gated $\mathrm{K}^{+}$channels $(\mathrm{Kv}(\mathrm{Ca}))$ or act as a second messenger to potentiate the activity of Kvs, we included $\mathrm{Ca}^{2+}$ channel blocker $\mathrm{CdCl}_{2}(20 \mu \mathrm{M})$ in the extracellular solution and fast $\mathrm{Ca}^{2}+$ buffer BAPTA $(30 \mathrm{mM})$ in the intracellular solution. Surprisingly, we found that these manipulations did not significantly affect the magnitude or time course of $\mathrm{KvF}$ as compared with the conditions where $\mathrm{Ca}^{2+}$ buffers were omitted or only a low concentration of slow $\mathrm{Ca}^{2+}$ buffer EGTA was present (Fig. 3). Our results indicate that facilitation of $I_{\mathrm{K}}$ is likely caused by use-dependent alterations in the intrinsic gating properties of Kvs, independent of VGCCs, $\mathrm{Kv}(\mathrm{Ca})$ or other $\mathrm{Ca}^{2+}$-dependent downstream signalling cascades.

Gating kinetics of Kvs encodes activity-induced facilitation. To explore the mechanisms underlying heterogeneity in $\mathrm{KvF}$, we did paired-pulse experiments in Chinese hamster ovary $(\mathrm{CHO})$ cell lines expressing recombinant homomeric Kv3.1 or heteromeric Kv1.1\&1.2 channels, which constituted the major subunits of native Kvs ${ }^{18,19,21-23}$ in the calyx of Held synapse. Simple spherical morphology of $\mathrm{CHO}$ cells also ensured accurate measurement of $I_{\mathrm{K}}$ with minimal space-clamp errors. We found that the PPF in Kv1.1\&1.2 currents was more robust (for example, PPF for Kv1.1\&1.2: $495.5 \pm 55.8 \%$ versus for Kv3.1: $389.4 \pm 31.8 \%$ at the interval of $1 \mathrm{~ms}$ ) and decayed much slower with increased inter-spike intervals than that in Kv3.1 currents ( $\tau=1.0 \mathrm{~ms}$ for Kv1.1\&1.2 versus $\tau=0.4 \mathrm{~ms}$ for Kv3.1; Fig. $4 \mathrm{a}-\mathrm{c}$ ), in line with the observations from native $I_{\mathrm{K}-\mathrm{HT}}$ and $I_{\mathrm{K}-\mathrm{LT}}$ in the nerve terminal (Fig. 1). Most notably, the temporal onset of $I_{\mathrm{K}}$ evoked by the second spikes was shifted towards to the early phase of the pseudo-spikes, as quantified by the time difference between the peak of APs and that of their evoked $I_{\mathrm{K}}$ (Fig. 4d). The timing shift of the second $I_{\mathrm{K}}$ declined exponentially over prolonged inter-spike intervals with the comparable time constants (Kv3.1: $0.4 \mathrm{~ms}$ versus Kv1.1\&1.2: $2.4 \mathrm{~ms}$; Fig. 4e,f) to those of PPF measured by the area integral of $I_{\mathrm{K}}$ (Fig. 4c). To investigate whether temperature has a role in $\mathrm{KvF}$, we applied similar paired-pulse paradigms at $35^{\circ} \mathrm{C}$ with briefer $\mathrm{AP}$ waveform and inter-spike intervals ( -90 to $100 \mathrm{mV}$, halfwidth: $0.15 / 0.3 \mathrm{~ms}$, intervals: $0.5-5 \mathrm{~ms}$ ) to mimic realistic temperature-dependent acceleration. Although it was evident that raising temperature speeded up activation and deactivation kinetics of these channels, we noted that PPF of $I_{\mathrm{K}}$ remained pronounced (for example, Kv1.1\&1.2: PPF $=256.2 \pm 28.0 \%$ at the interval of $1 \mathrm{~ms}$; Supplementary Fig. 5). These results reinforce the notion that Kvs facilitate during repetitive activity regardless of their subtypes or experimental temperature.

In many cases, $I_{K}$ evoked by the second spikes at the short intervals showed two components with clear kinks on their rise phase (Figs 1-4). We hypothesized that activated Kvs by the first spikes may appear closed as indicated by a complete return of $I_{\mathrm{K}}$ to the baseline, but actually have not recovered to the resting close state, leaving a subpopulation of Kvs in the intermediate gating states that are more willing to open. Therefore, the second spikes would result in larger $I_{\mathrm{K}}$ with shorter activation latencies. To address this possibility, we used depolarization steps to measure voltage-dependent activation of Kv3.1 and Kv1.1\&1.2 from $\mathrm{CHO}$ cells with or without a short preceding 'priming' spike at 22 or $35^{\circ} \mathrm{C}$ (Fig. 4g,h and Supplementary Fig. 5). When the amplitude of $I_{\mathrm{K}}$ was plotted against the depolarizing voltages, we noticed an early onset of $I_{\mathrm{K}}$ with a lower apparent activation threshold for the 'priming' groups, leading to a global left shift in their current $(I)$-voltage $(V)$ curves at both temperatures (Fig. 4i,j and Supplementary Fig. 5). These observations provided direct evidence for the predictions made by previous studies ${ }^{25,26}$, which examined the effects of long-step prepulses on the gating currents of Shaker $\mathrm{K}^{+}$channels in cut-open oocytes, and implicated that intrinsic gating of $\mathrm{K}^{+}$channels might underlie the 'Cole-Moore effect' as first described in the squid axon ${ }^{27}$. We propose that this left shift in the I-V relationships by preceding AP-like activity increases the open probability of Kvs to subsequent stimuli, which gives rise to the prominent facilitation of $I_{\mathrm{K}}$ in response to paired pulses or high-frequency AP bursts.

Given that both native and recombinant Kvs exhibit activitydependent facilitation, we postulated that this functional phenotype could reside in intrinsic gating kinetics of these channels. To test this, we first fit activation and deactivation kinetics of $I_{\mathrm{K}-\mathrm{HT}}$ and $I_{\mathrm{K}-\mathrm{LT}}$ recorded from the outside-out calyceal patches with the Markov modelling to derive gating rate 
a

Real AP train at $400 \mathrm{~Hz}$ (voltage command)

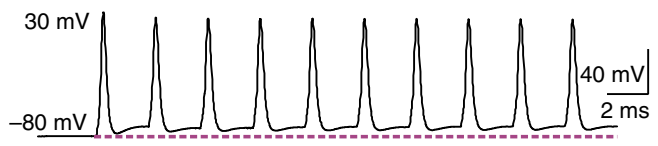

$I_{\mathrm{K}}$ recorded in no $\mathrm{Ca}^{2+}$ buffer

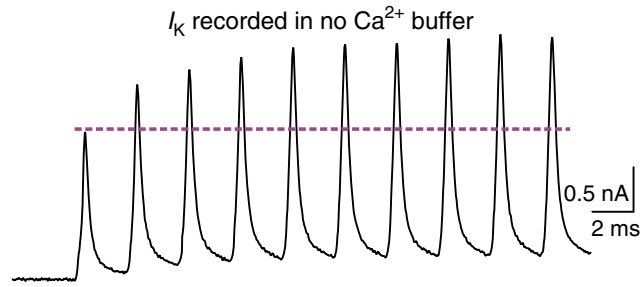

$I_{\mathrm{K}}$ recorded in EGTA $(0.5 \mathrm{mM})$

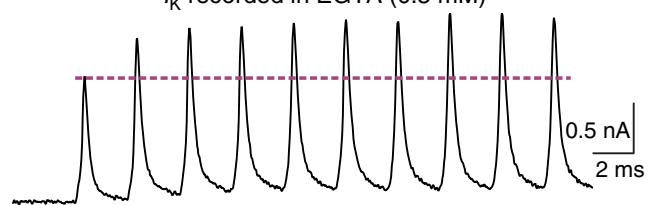

$I_{\mathrm{K}}$ recorded in BAPTA $(30 \mathrm{mM})$

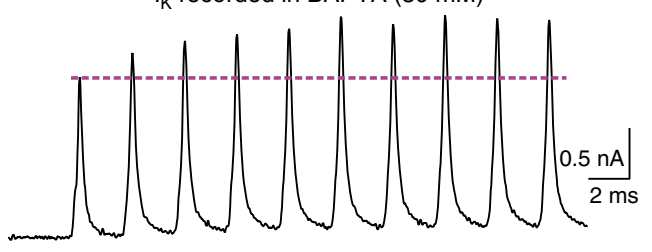

b $160 \quad I_{\mathrm{K}}$ recorded in no $\mathrm{Ca}^{2+}$ buffer
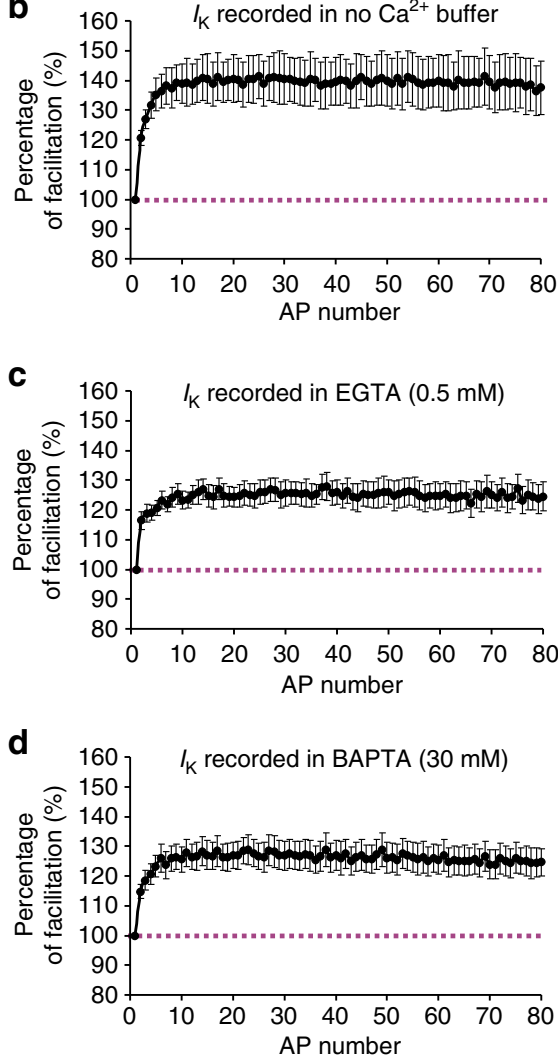

Figure 3 | Activity-dependent facilitation of $\mathbf{I}_{\mathbf{K}}$ is $\mathbf{C a}^{\mathbf{2}}+$ independent. (a) Whole-cell recordings of $I_{\mathbf{K}}$ evoked by a typical presynaptic AP trains (previously obtained from the calyx of Held nerve terminal, the 1st AP has an amplitude of $110 \mathrm{mV}$, half-width of $0.28 \mathrm{~ms}$ and depolarizing after potential (DAP) of

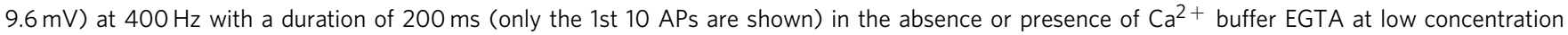
$(0.5 \mathrm{mM})$ or BAPTA at high concentration $(30 \mathrm{mM})$. (b-d) The normalized amplitude of $I_{\mathrm{K}}$ (to the 1st response in a train) is summarized for no-Ca ${ }^{2+}$ buffer $(n=4, \mathbf{b}), \operatorname{EGTA}(n=6, \mathbf{c})$ and $\operatorname{BAPTA}(n=5, \mathbf{d})$ groups. Error bars indicate \pm s.e.m. 1 st, first.

constants ${ }^{14-16}$. We found that the classic six-state linear gating scheme $\left(\mathrm{C}_{0}, \mathrm{C}_{1}, \mathrm{C}_{2}, \mathrm{C}_{3}, \mathrm{C}_{4}\right.$ and $\left.\mathrm{O}\right)$ fit experimental data well, and more importantly recapitulated the major characteristics of these native currents. For instance, $I_{\mathrm{K} \text {-LT }}$ predicted by the model closely resembled the recorded currents in the lower activation threshold of $I_{\mathrm{K}}$ following a preceding spike and paired-pulse-induced facilitation (Fig. 5a-c). Model fittings revealed that the backward transition rate constant $(\beta)$ of K-LT was significantly slower than that of K-HT, whereas the forward rate constants $(\alpha)$ for both subtypes of Kvs were comparable (Table 1), indicating that slower return from the intermediate closed states $\left(\mathrm{C}_{4} \ldots \mathrm{C}_{1}\right)$ to resting closed state $\left(\mathrm{C}_{0}\right)$ may be associated with more robust facilitation of $I_{\mathrm{K}-\mathrm{LT}}$. Indeed, when we plotted probabilities of all the kinetic states at equilibrium before the first spikes during the pairedpulse paradigm, we found that K-LT largely stayed in the $\mathrm{C}_{0}$ and $\mathrm{C}_{1}$ states with the other states being less dominant. The same analysis at the onset of the second pulses showed that the probability for the $\mathrm{C}_{4}$ or $\mathrm{O}$ state of K-LT was very low and the $\mathrm{C}_{1}$ state remained relatively constant at varied inter-pulse intervals. By contrast, the distribution of other closed states was highly sensitive to the intervals. As the interval was shortened (for example, $<5 \mathrm{~ms}$ ), the probability of the channel remaining in the intermediate states $\left(\mathrm{C}_{2}\right.$ and $\left.\mathrm{C}_{3}\right)$ steeply increased at the expense of lowering the probability for the resting closed state $\left(\mathrm{C}_{0}\right)$. This marked re-distribution of different closed states well correlated with the time course of $I_{\mathrm{K}-\mathrm{LT}}$ facilitation (Fig. $5 \mathrm{c}$ ). Taken together, we interpret our experimental and simulation results as such that the intermediate closed states of Kvs arising from previous activity decrease energy barrier to reach the open state, and hence account for accelerated activation and lowered activation threshold for Kvs susceptible to subsequent stimuli.

KvF reduces AP amplitude and plateau potential of AP trains. Lacking ideal blockers, which only inhibit activity-dependent facilitation of Kvs without affecting their basal properties, precluded us from pharmacologically examining the physiological roles of $\mathrm{KvF}$ in high-frequency spiking. To overcome this difficulty, we performed computer simulations by creating a modified Hodgkin-Huxley $(\mathrm{H}-\mathrm{H})^{1}$ model cell (capacitance $6 \mathrm{pF}$ and resting potential $-80 \mathrm{mV}$ ) containing four components (that is, potassium conductance $\left(g_{\mathrm{K}-\mathrm{HT}}\right.$ and $\left.g_{\mathrm{K}-\mathrm{LT}}\right)$, leak conductance $\left(g_{\mathrm{L}}\right)$ and sodium conductance $\left(g_{\mathrm{Na}}\right.$; Fig. $\left.6 \mathrm{a}, \mathrm{b}\right)$, where $g_{\mathrm{K}-\mathrm{HT}}, g_{\mathrm{K}-\mathrm{LT}}$, $g_{\mathrm{L}}$ and $g_{\mathrm{Na}}$ were free variables and APs were initiated by a brief inward square current $(2 \mathrm{nA})^{16,28}$. The gating kinetics of $g_{\mathrm{Na}}$ was established by fitting activation, deactivation and inactivation of $I_{\mathrm{Na}}$ obtained from the outside-out patches near the heminode region of calyceal terminals (Supplementary Fig. 6). Subsequently, we incorporated this conductance along with two components of Kvs derived from the Markov model into the modified $\mathrm{H}-\mathrm{H}$ model cell, and produced AP trains that had the similar waveform to those realistic spikes recorded from the nerve terminal and as well as frequency-dependent adaptation $(200-600 \mathrm{~Hz}$; Figs $6 \mathrm{~b}$ and $7 \mathrm{a}-\mathrm{c})$. Figure $6 \mathrm{~b}$ showed a simulated $400 \mathrm{~Hz}$ train, during which $I_{\mathrm{K}-\mathrm{LT}}$ but not $I_{\mathrm{K}-\mathrm{HT}}$ facilitated greatly, consistent with the experimental evidence of their distinct decay time courses for PPF (Fig. 1e,f). As revealed by our model (Fig. 5), fast deactivation kinetics of $I_{\mathrm{K}-\mathrm{HT}}$ might account for the lack of 
a

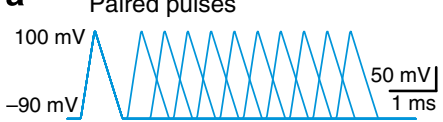

$-90 \mathrm{mV}$

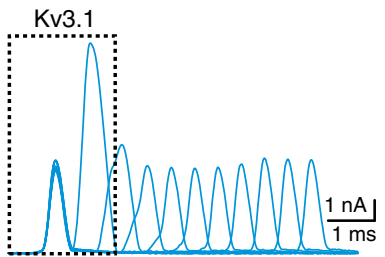

d Zoom in

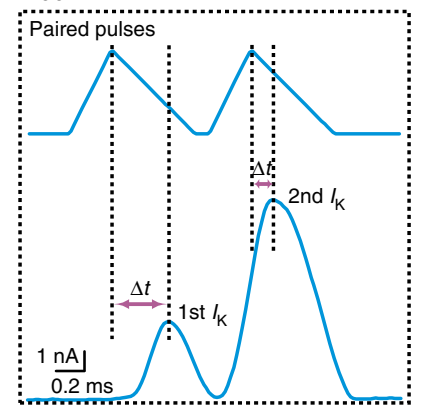

g

Stimulation protocol (no priming)
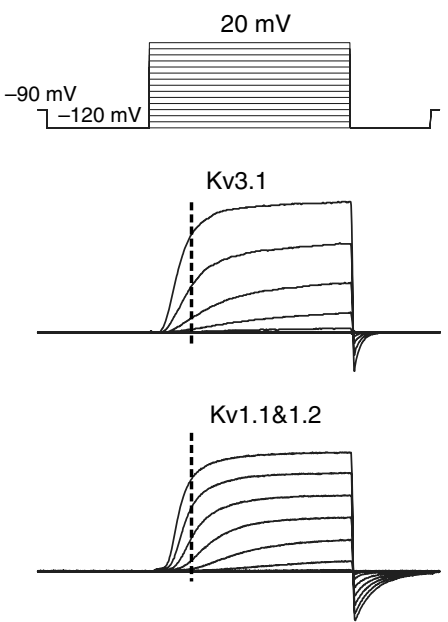

b Paired pulses
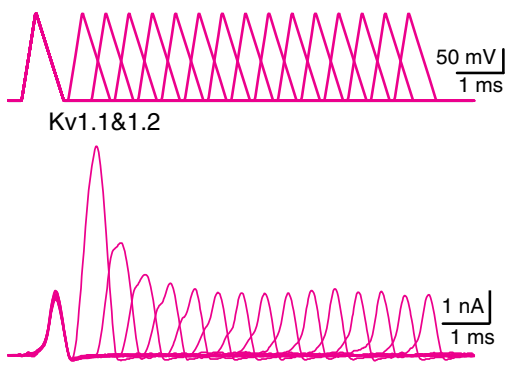

e $\quad \mathrm{Kv} 3.1$

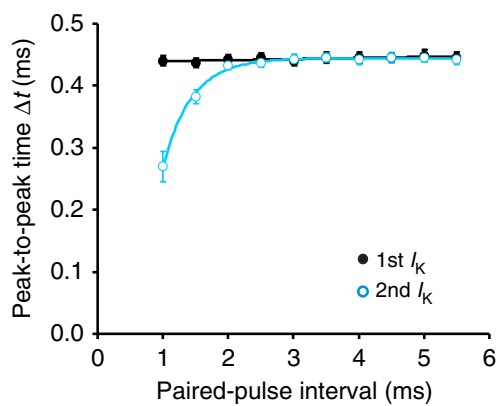

h

Stimulation protocol (with priming)
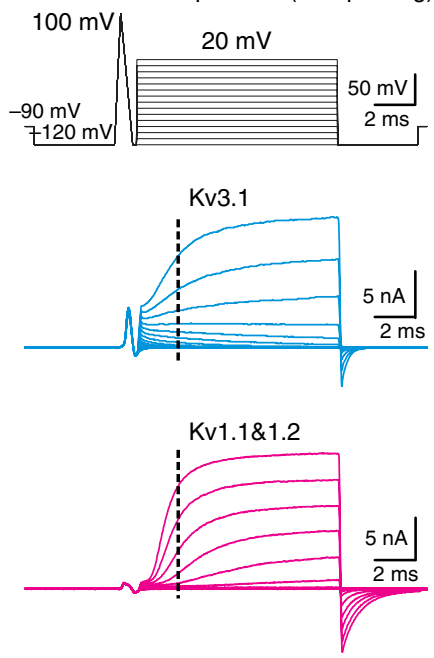

C By area integral of $I_{\mathrm{K}}$

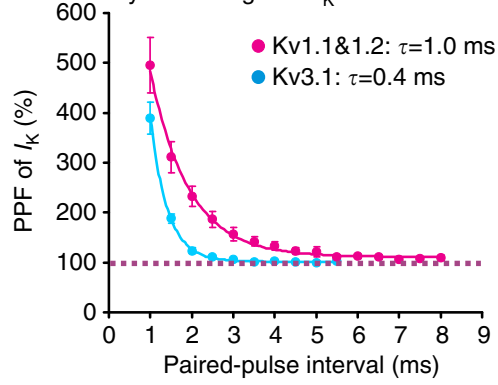

f $\quad \mathrm{Kv} 1.1 \& 1.2$

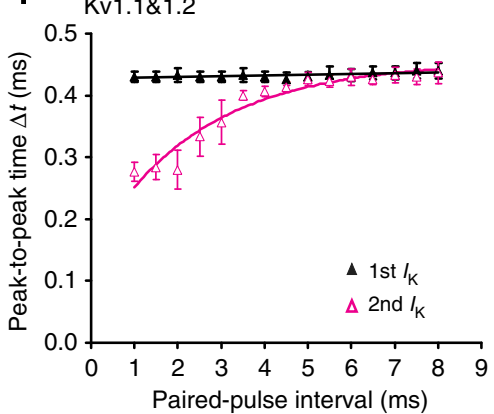

i

Kv3.1

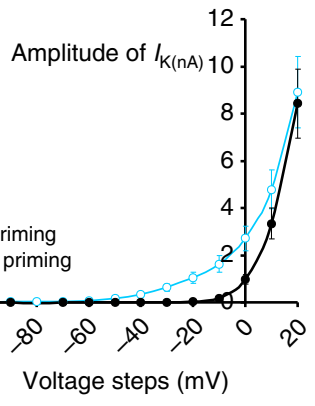

j

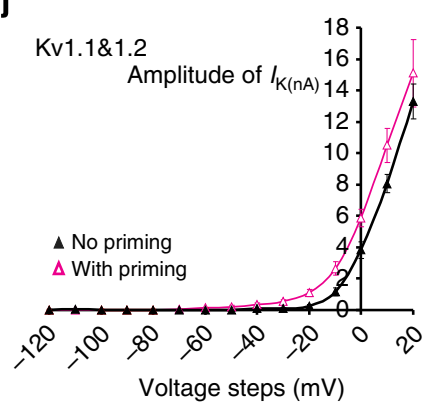

Figure 4 | Mechanisms underlying KvF from recombinant Kv channels. (a,b) $I_{K}$ (bottom panels) generated from CHO cells expressing Kv3.1 (blue, a) or Kv1.1\&1.2 channels (magenta, b) by paired-pulse stimulation protocol (AP waveform: -90 to $+100 \mathrm{mV}$, rise time: $0.3 \mathrm{~ms}$, decay time: $0.6 \mathrm{~ms}$, pairedpulse interval: $1-8 \mathrm{~ms}$ with increment of $0.5 \mathrm{~ms}$, top panels). (c) PPF in the area integral of $I_{\mathrm{K}}$ is summarized for CHO cells transfected with $\mathrm{Kv} 3.1$ (blue circles, $n=8$ ) or Kv1.1\&1.2 (magenta circles, $n=6$ ) constructs. The solid lines are fits to a single exponential function and time constants are shown in the figure. (d-f) The 1st pair of $I_{K}$ in $\mathbf{a}$ are focused to show the time difference $(\Delta t)$ between the peak of APs and that of corresponding $I_{K}(\mathbf{d})$. Note the marked early onset of the $2 n d I_{K}$ compared to the $1 s t I_{K}$. Quantitative analysis of $\Delta t$ are demonstrated for expression of Kv3.1 ( $n=8$, e) or Kv1.1\&1.2 $(n=6, \mathbf{f})$. (g-j) Voltage steps from -120 to $20 \mathrm{mV}$ ( $10 \mathrm{~ms}$ long, top panels) applied to elicit $I_{\mathrm{K}}$ from a CHO cell expressing Kv3.1 (middle panels) or Kv1.1\&1.2 (bottom panels) with $(\mathbf{h})$ or without $(\mathbf{g})$ the pre-pulse $(-120-100 \mathrm{mV}$, rise time: $0.3 \mathrm{~ms}$, decay time: $0.6 \mathrm{~ms})$. The amplitude of $I_{\mathrm{K}}$ at varied potentials, measured at $2 \mathrm{~ms}$ after beginning of depolarization steps (dotted lines), is plotted in $\mathbf{i}(\mathrm{Kv} 3.1, n=4)$ and $\mathbf{j}(\mathrm{Kv} 1.1 \& 1.2, n=4)$. Error bars indicate \pm s.e.m. 1st, first; 2 nd, second.

facilitation, particularly when evoked by a real AP train with usedependent reduction in the amplitude. To specifically delineate the function of facilitation of $I_{\mathrm{K}-\mathrm{LT}}$, we manually adjusted the deactivation rate constant $\beta$ (Table 2: parameter $c$ ) until activitydependent facilitation was largely removed while the amplitude of the first $I_{\mathrm{K}-\mathrm{LT}}$ was maintained by increasing its total conductance. Under these conditions, we disclosed that attenuation of $I_{\mathrm{K}-\mathrm{LT}}$ facilitation eliminated activity-dependent reduction in the spike amplitude and elevated the plateau potential in the AP train (Fig. 6b). Surprisingly, $I_{\mathrm{Na}}$ derived from the model cell with 
a
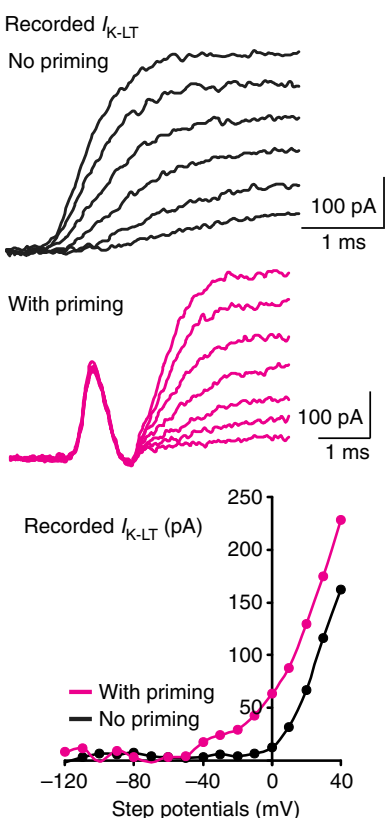

b
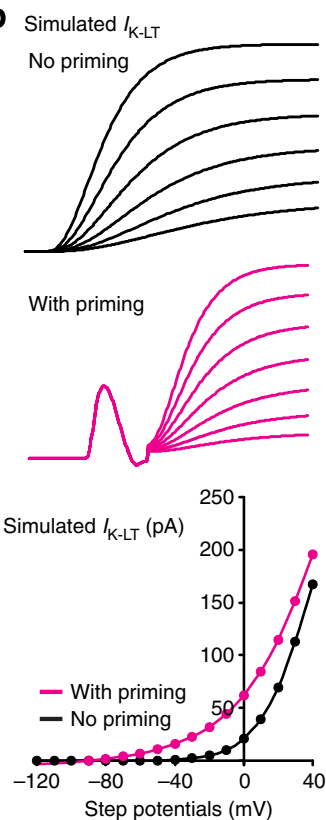

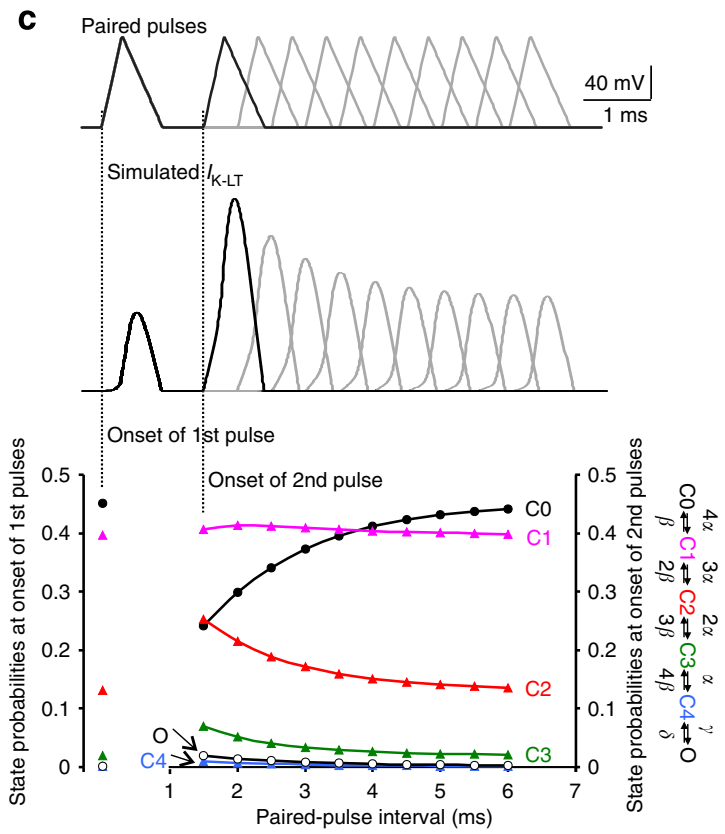

Figure 5 | Computer simulation of gating kinetics of Kvs. $(\mathbf{a}, \mathbf{b}) I_{K}$ recorded from an outside-out patch of the calyx of Held nerve terminal (a) or simulated by a computational model containing five closed states and one open state (b) in response to depolarization steps from -120 to $40 \mathrm{mV}$ with (magenta traces, middle panels) or without (black traces, top panels) a preceding AP-like ramp (amplitude: $110 \mathrm{mV}$; half-width: $0.45 \mathrm{~ms}$ ). The amplitude of $I_{\mathrm{K}}$ at $2 \mathrm{~ms}$ after the start of voltage steps is plotted against step potentials (bottom panels) to illustrate the early activation of Kvs by the preceding depolarization. (c) Example of simulated $I_{\mathrm{K}}$ (middle) in response to a paired-pulse protocol (spike amplitude: $110 \mathrm{mV}$ and half-width: $0.45 \mathrm{~ms}$, paired-pulse interval: 1.5 $6 \mathrm{~ms}$; top). The magnitude and frequency-dependence of KvF are well reminiscent of experimental recordings of $I_{\mathrm{K}}$ as shown in Fig. 1. Based on the simulation model (Table 1) with all the six states summated to 1 at any given time point during the paired-pulse paradigm, probabilities for each state of K-LT at the onset of the 1st or 2nd spikes are plotted against various inter-pulse intervals (bottom). 1st, first; 2nd, second.

Table 1 | Computational simulation of Kvs.

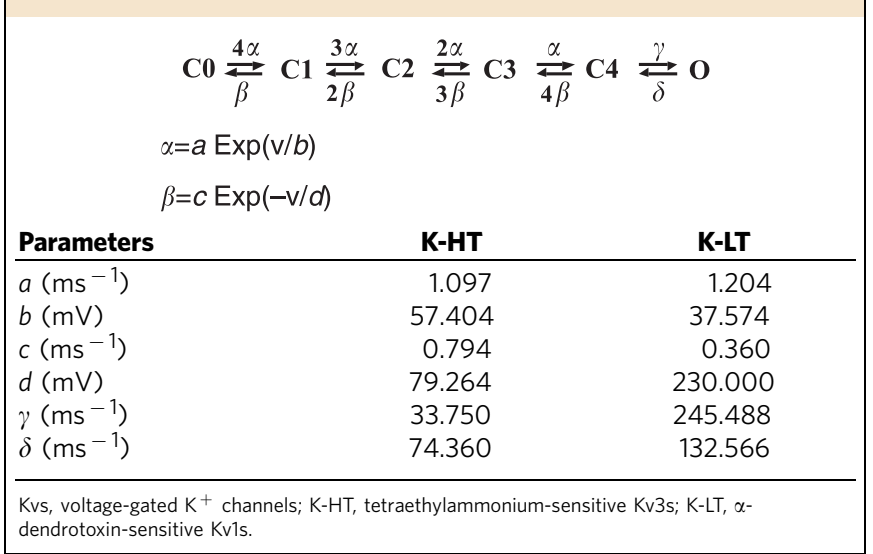

minimal $I_{\mathrm{K}-\mathrm{LT}}$ facilitation showed more prominent use-dependent inactivation, and yet remained sufficient to drive the spikes to higher amplitude. Activity-dependent decrease in the spike amplitude has classically been viewed as a result of $\mathrm{Na}^{+}$ channel inactivation $2,7,11$; however, the last line of our simulation results suggests that $I_{\mathrm{K}-\mathrm{LT}}$ facilitation can have an active role in regulating the AP amplitude by counteracting $I_{\mathrm{Na}}$ during repetitive activity.

KvF modulates short-term synaptic plasticity. To elucidate functional consequences of activity-induced facilitation of Kvs, we recorded presynaptic $\mathrm{Ca}^{2}+$ currents $\left(I_{\mathrm{Ca}}\right)$ from the calyx of Held nerve terminal by applying the simulated presynaptic spike trains as voltage-clamp command templates with normal or attenuated $I_{\mathrm{K}-\mathrm{LT}}$ facilitation. We found that attenuation of $\mathrm{KvF}$ had little effect on the amplitude of $I_{\mathrm{Ca}}$ by the first APs, but significantly enhanced $I_{\mathrm{Ca}}$ and $\mathrm{Ca}^{2+}$-dependent facilitation of $I_{\mathrm{Ca}}$ by subsequent APs in a frequency-dependent manner (Fig. 7a-d). Most strikingly, it converted depression of $I_{\mathrm{Ca}}$ to potentiation at 400 or $600 \mathrm{~Hz}$, demonstrating a profound impact of $I_{\mathrm{K}-\mathrm{LT}}$ facilitation on the magnitude and time course of short-term plasticity in $I_{\mathrm{Ca}}$. Given that quantal output of any given synapse is a power function of $I_{\mathrm{Ca}}{ }^{29-31}$, one can readily predict that the amount of transmitter release would be markedly affected. To this end, we made simultaneous paired recordings of $I_{\mathrm{Ca}}$ and excitatory postsynaptic currents ( $I_{\mathrm{EPSC}}$ ) from the calyx of Held synapse. When the AP template with normal $I_{\mathrm{K}-\mathrm{LT}}$ facilitation was repeatedly applied to the calyces, we found that short-term depression (STD) in $\mathrm{I}_{\mathrm{EPSC}}$ dominated at $400 \mathrm{~Hz}$, consistent with previous observations from this synapse in response to afferent stimulation $^{32}$. By contrast, when the template with attenuated $I_{\mathrm{K}}$ LT facilitation was delivered to the same nerve terminals, we noted substantial short-term potentiation (STP) of $I_{\mathrm{EPSC}}$ in the early phase of the train stimulation (maximal potentiation: $115.8 \pm 6.2 \%$ ), followed by a more profound and rapid STD while the first $\mathrm{I}_{\mathrm{EPSC}}$ for both sets of AP templates were not different (decay time constant of STD: $22.1 \pm 3.9$ versus $7.9 \pm 0.5 \mathrm{~ms}$ for normal and attenuated $I_{\mathrm{K}-\mathrm{LT}}$ facilitation, respectively; Fig. 7c,d). Unlike highly plastic synapses with heterogeneous release probability ${ }^{33}$, trail-to-trail fluctuations in $I_{\text {EPSC }}$ evoked by the same stimulation templates at the calyx of Held synapse were small (Fig. 7c) as a consequence of a relatively large number of release sites and size of the readily releasable pool (RRP) of synaptic vesicles $(\mathrm{SVs})^{17}$. In addition to increased AP 


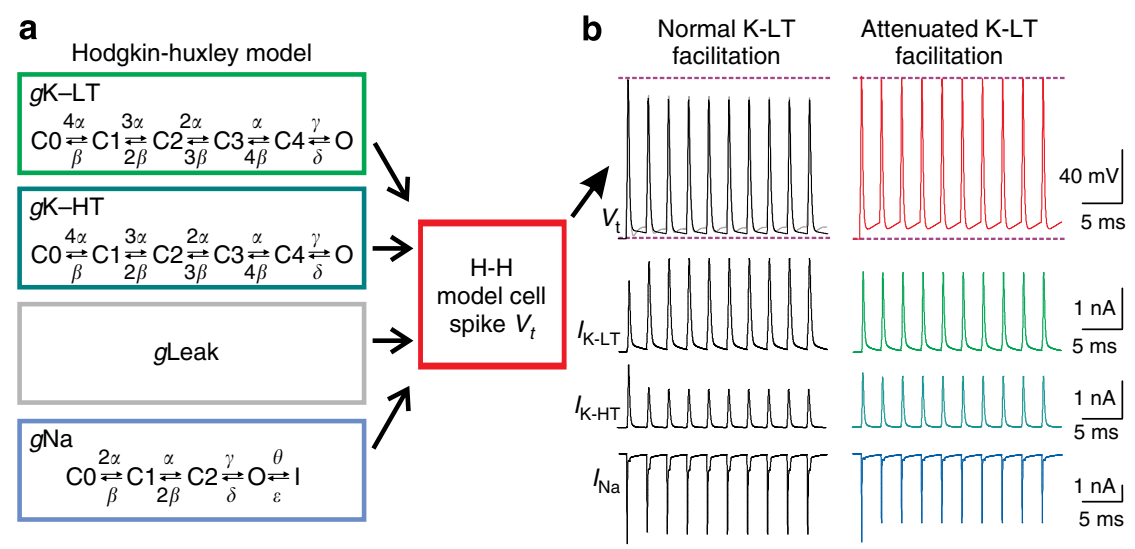

Figure 6 | Specific contribution of KvF to AP trains in a modified H-H model cell. (a) A modified Hodgkin-Huxley (H-H) model containing four components, that is, potassium (both low- ( $g \mathrm{~K}-\mathrm{LT}$ ) and high-threshold ( $g \mathrm{~K}-\mathrm{HT})$ ), leak ( $g$ Leak) and sodium ( $g \mathrm{Na}$ ) conductance, where $g \mathrm{~K}-\mathrm{LT}$, $g \mathrm{~K}-\mathrm{HT}$, $g$ Leak and $g \mathrm{Na}$ are free variables while APs are evoked by brief current injections ( $2 \mathrm{nA}, 0.2 \mathrm{~ms}$ ). (b) Simulated AP trains at $400 \mathrm{~Hz}$ (top panels) before (left) and after (right) attenuating facilitation of $I_{\mathrm{K}-\mathrm{LT}}$ by manipulating a single parameter $c$ in the rate constant $\beta$ (Table 2). The underlying currents for these two different patterns of APs are shown in the following panels. A recorded realistic AP train (grey) is included in the top left panel to illustrate its close resemblance to the simulated one. Note that attenuation of $I_{K-L T}$ facilitation increases the spike amplitude and interspike potential.

amplitude after the first spike when $I_{\mathrm{K}-\mathrm{LT}}$ facilitation was attenuated, elevated plateau potential between spikes might have also amplified the effects of higher residual $\mathrm{Ca}^{2}+$ buildup and accelerated STD, as suggested by the previous studies showing a strong impact of small depolarization on the quantal output $^{34-36}$. Taken together, our results demonstrate that these seemingly subtle changes in spike amplitude and plateau potential due to $\mathrm{KvF}$ converge to effectively constrain $\mathrm{Ca}^{2+}$ influx and residual accumulation of $\mathrm{Ca}^{2+}$ during AP bursts, leading to significant alterations in the polarity and magnitude of shortterm synaptic plasticity. Given the finite number of SVs in the $\mathrm{RRP}$ at central synapses, we suggest that presynaptic KvF may be important for effectively conserving the RRP to sustain adequate quantal output during high-frequency neurotransmission.

Presynaptic KvF promotes high-fidelity of postsynaptic spiking. To directly test the physiological significance of $\mathrm{KvF}$ observed from the calyx of Held nerve terminal, we made dynamic-clamp recordings to examine the firing patterns of their postsynaptic neurons in response to two sets of conductance templates (STD-only versus STP + STD), derived from native $I_{\text {EPSC }}$ elicited at $400 \mathrm{~Hz}$ with normal or attenuated $I_{\mathrm{K}-\mathrm{LT}}$ facilitation (Fig. 7c). We first generated such digital templates by converting $I_{\text {EPSC }}$ into synaptic conductance (Gsyn) and then directly fed them into the postsynaptic neurons via patch electrodes. These conductances were reminiscent of realistic synaptic inputs with trial-to-trail fluctuations in the respective cases where $I_{\mathrm{K}-\mathrm{LT}}$ facilitation was present or largely absent. Notwithstanding the variability among five individual sweeps within the same groups, on average the STD-only and STP + STD templates produced distinct firing patterns (Fig. 8a-d). When the STD-only templates were applied, spikes were evoked reliably by the first eight stimuli before failures appeared in an alternating manner (Fig. 8e,h; spike failure rate: $36.7 \pm 2.4 \%$ ), typical of the phase-locking phenotype of these native neurons ${ }^{20}$. In contrast, when stimulated with the STP + STD templates, the same neurons fired much fewer consecutive spikes early in the train and subsequently failed to generate spikes completely (Fig. 8f,h; spike failure rate: $52.9 \pm 1.6 \%)$. Quantitative analyses of the time difference between the peak of the seventh APs and that of the seventh current inputs showed a substantial delay in the spike latency for the STP + STD paradigm $(0.95 \pm 0.06 \mathrm{~ms})$ as compared with the STD-only template $(0.72 \pm 0.02 \mathrm{~ms}$; Fig. 8g,i). Collectively, this evidence denotes that facilitation of presynaptic $I_{\mathrm{K}}$ may serve as a built-in mechanism to support sustained postsynaptic spiking by dynamically attenuating presynaptic $\mathrm{Ca}^{2}+$ influx and preventing rapid depletion of the RRP during AP bursts.

\section{Discussion}

Our study has established a new form of frequency-dependent but $\mathrm{Ca}^{2+}$-independent facilitation of Kvs, which is widely present in neurons from hippocampus, cerebellum and auditory brainstem. Particularly relevant to the physiological functionality of fast synapses such as the calyx of Held synapse, facilitation of presynaptic $I_{\mathrm{K}-\mathrm{LT}}$ attenuates the spike amplitude and inter-spike potential during high-frequency bursts. It impacts the magnitude and polarity of short-term plasticity in presynaptic $\mathrm{Ca}^{2+}$ inflow and transmitter release, ultimately enhancing the fidelity of postsynaptic spiking (Figs 7 and 8). Given a limited size of the RRP in the nerve terminal, we suggest that frequency-dependent attenuation in spike amplitude and inter-spike potential by facilitating Kvs is an indispensable adaptation for the efficient usage of release-competent SVs to maintain sustained synaptic drive. Such a mechanism enhances the phase-locking phenotype of the calyx of Held synapse, which is critical for sound localization $^{20}$. Extrapolating from this proof of principle at the calyx of Held synapse, we suggest that similar frequencydependent facilitation of Kvs at the axons or boutons of other fast-spiking central neurons may restrain depletion of SVs in the RRP and help improve sustainability of neurotransmission at high-frequency neural activity.

Unlike $\mathrm{Ca}^{2+}$-dependent facilitation of VGCCs through calmodulin binding to VGCCs or facilitation of $\mathrm{Kv}(\mathrm{Ca})$ by $\mathrm{Ca}^{2+}$ binding to their own EF motifs ${ }^{37,38}$, we have found that facilitation of Kvs exclusively results from the intrinsic gating kinetics of these channels in transition between the intermediate closed states and the open state during repetitive activity. Similar features of Kvs from several native neurons and mammalian cell lines expressing recombinant Kv1.1\&1.2 or Kv3.1 channels reinforce the view that Kvs express facilitation without other endogenous modulators, despite that low- and high-threshold Kvs bear different extent and dynamics of facilitation. 
a

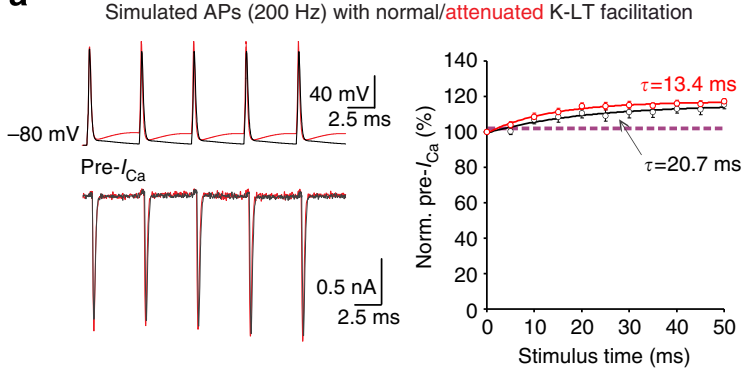

b

Simulated APs $(600 \mathrm{~Hz})$ with normal/attenuated K-LT facilitation
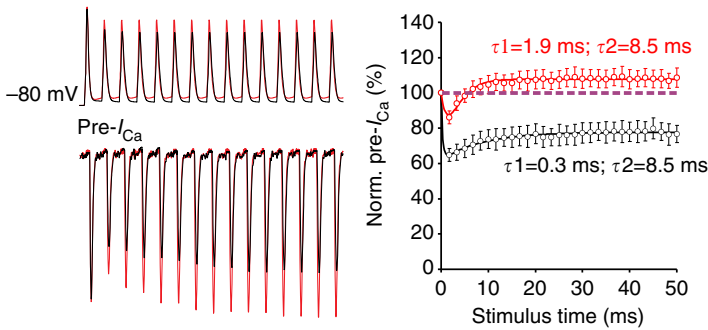

C

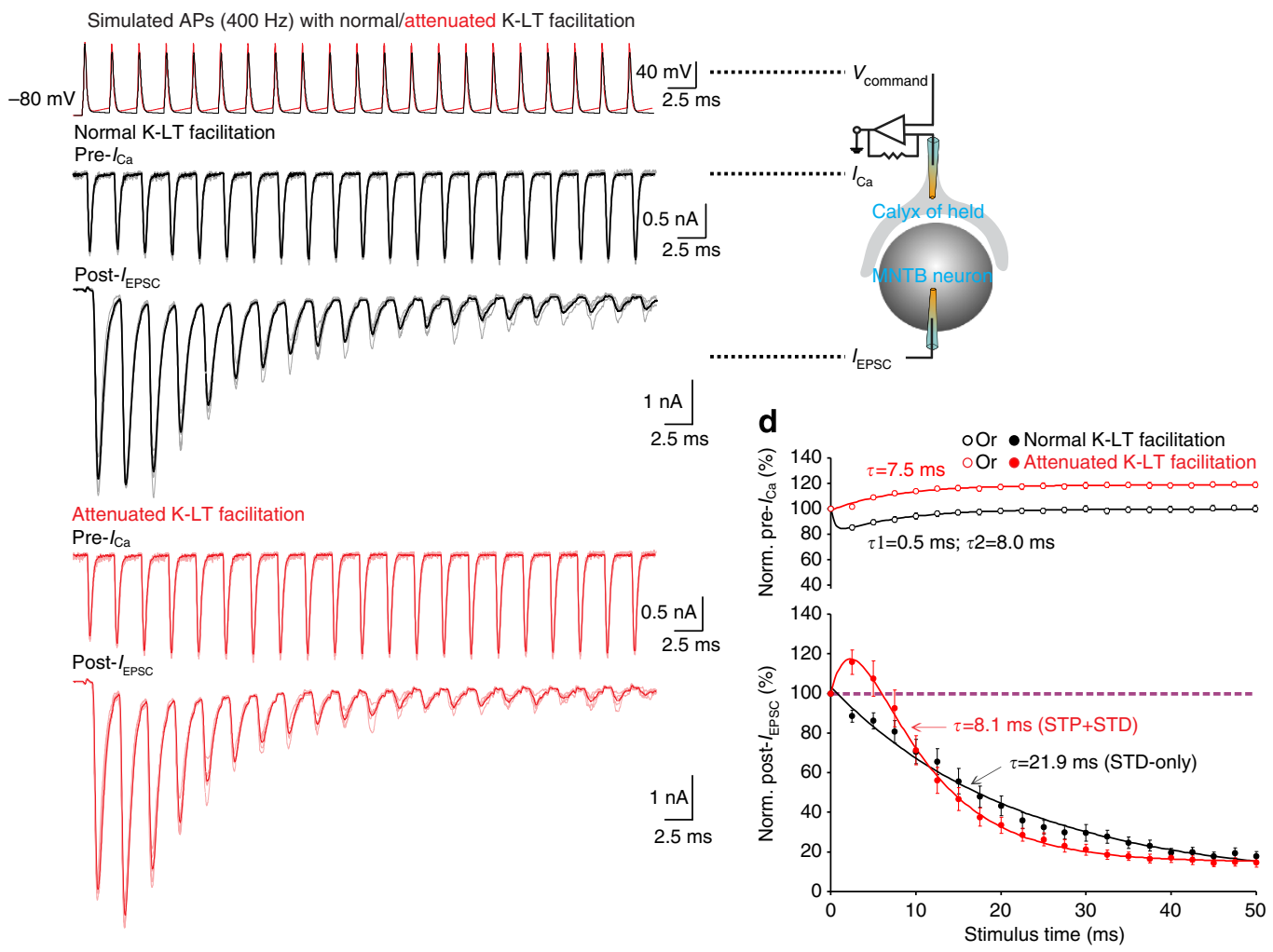

Figure 7 | Strong impact of KvF on synaptic input-output relationship. (a,b) Representative recordings of presynaptic $\mathrm{Ca}^{2+} \mathrm{currents}\left(\mathrm{pre}-\mathrm{I}_{\mathrm{Ca}}\right.$, bottom) from the calyces elicited by simulated AP trains (top) at $200 \mathrm{~Hz}$ (a) or $600 \mathrm{~Hz}$ (b) before (black traces), and after (red traces) attenuating K-LT facilitation. The amplitude of pre- $I_{\mathrm{Ca}}$ is normalized (Norm.) to that of the 1 st pre- $I_{\mathrm{Ca}}$ during the train stimuli and plotted against stimulus time (the time for the 1 st $\mathrm{AP}$ stimulation is set as zero). The solid lines in the right panels are fits to a single or double exponential function and the time constants are given ( $n=9$ for $200 \mathrm{~Hz}$ and $n=6$ for $600 \mathrm{~Hz}$ group). (c) Simultaneous recordings of pre- $I_{\mathrm{Ca}}$ (middle) and excitatory postsynaptic currents (post- $I_{\mathrm{EPSC}}$, bottom) from the calyx of Held synapse repeatedly evoked by simulated spikes at $400 \mathrm{~Hz}$ (top) with normal (grey traces) or attenuated (pink traces) K-LT facilitation. Average traces of five repeats for each group are highlighted in black or red. Recording configuration is depicted on the right. (d) The amplitude of pre- $I_{\mathrm{Ca}}$ (top) over $400 \mathrm{~Hz}$ train stimuli is fitted with a single/dual exponential function as shown with the solid lines. The post-IEPSC (bottom) are described by a single (black line, with normal K-LT facilitation) or double (red line, with attenuated K-LT facilitation) exponential function ( $n=8$ for each group). The amplitude of both pre- $I_{\mathrm{Ca}}$ and post- $\mathrm{I}_{\text {EPSC }}$ are normalized to that of the 1 st response in a train and plotted against stimulus time. Error bars indicate \pm s.e.m. 1st, first.

Table 2 | Kinetic parameters for simulating spike trains using the modified H-H model with normal or attenuated K-LT facilitation.

$\alpha=1.2 \operatorname{Exp}(v / 37.6) \beta=c \operatorname{Exp}(-v / 230.0)$

Normal K-LT facilitation

Attenuated K-LT facilitation
Parameter $\mathrm{c}$ in $\beta\left(\mathrm{ms}^{-1}\right)$

0.36

0.8 g K-LT $_{\text {(nS) }}$

394

670

$\boldsymbol{g}_{\mathbf{K}-\mathbf{H T}}(\mathbf{n S})$
3,500

2,500

$g_{\text {Leak }}(\mathrm{nS})$

8

8
8 $I_{\text {Stim }}(\mathbf{n A})$

$\mathrm{H}-\mathrm{H}$, Hodgkin-Huxley; gK-LT, potassium conductance (low threshold); gK-HT, potassium conductance (high-threshold); gLeak, leak conductance; $g \mathrm{Na}$, sodium conductance. 
a

Postsynaptic APs evoked by STD-only EPSC templates $(400 \mathrm{~Hz})$ with normal K-LT facilitation

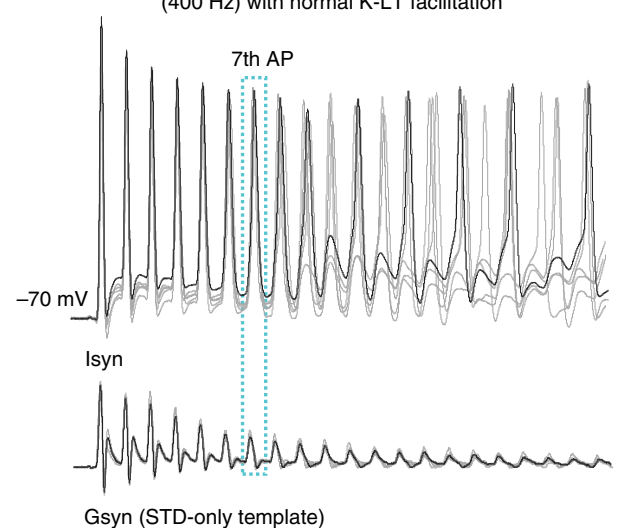

MAcmonnmunn

C

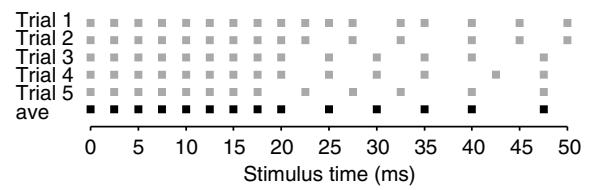

e

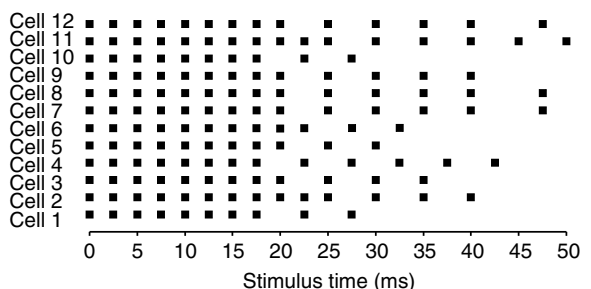

b Postsynaptic APs evoked by STP+STD EPSC templates $(400 \mathrm{~Hz})$ with attenuated K-LT facilitation
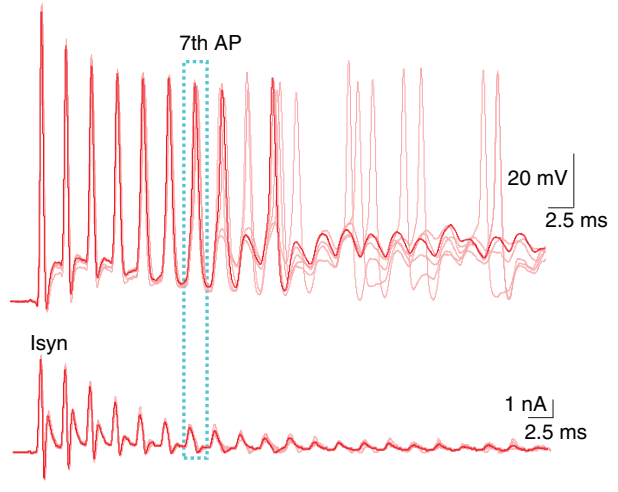

Gsyn (STP+STD template)

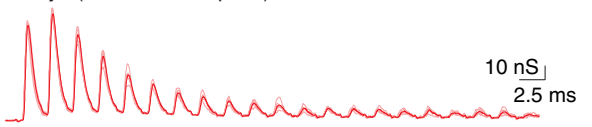

d

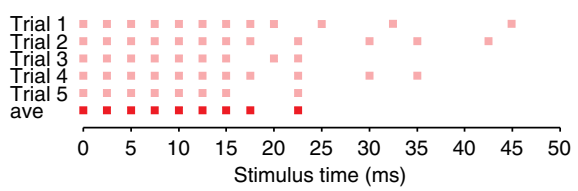

f

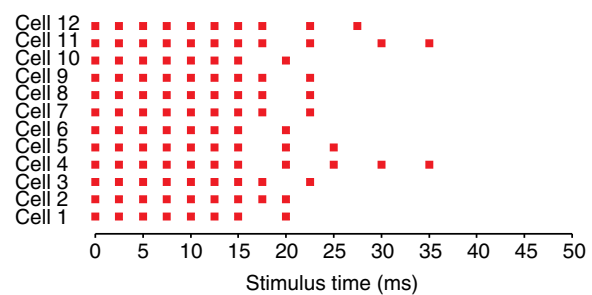

g

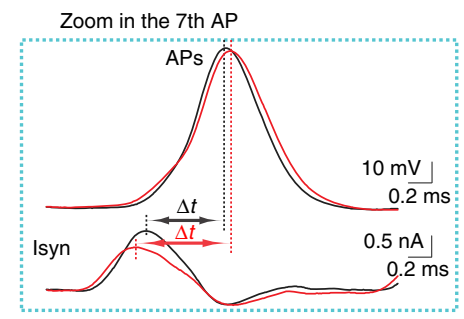

h $\mathbf{n}$ Normal K-LT facilitation - Attenuated K-LT facilitation

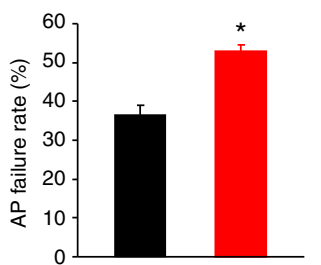

I Normal K-LT facilitation - Attenuated K-LT facilitation

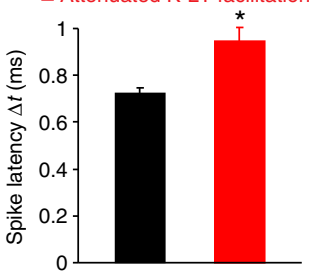

Figure 8 | Indispensable role of KvF in boosting spiking fidelity of principal neurons. (a,b) Conductance templates (Gsyn), STD-only (individual trials in grey and average (Ave) trial in black, a) and STP + STD (individual trials in pink and average trial in red, b) are generated by reversing the polarity of two sets of representative recordings of post-IEPSC, respectively, evoked by the train stimulation $(400 \mathrm{~Hz})$ with normal or attenuated $I_{\mathrm{K}-\mathrm{LT}}$ facilitation (bottom panels). Spikes evoked by stimulation currents (Isyn, middle panels) in the dynamic-clamp configuration by injecting the EPSC templates into the same postsynaptic neurons are shown in top panels. (c,d) APs produced by the five individual and average conductance templates for each group are summarized. Each square represents a spike. (e,f) Raster plots of APs generated by the average conductances of five repeats against stimulus time in 12 cells. (g) Time difference between the peak of the 7th stimulation currents and that of their resulting APs during the train stimuli, termed as spike latency, is illustrated to display the prolonged spike latency without $I_{\mathrm{K}-L T}$ facilitation. $(\mathbf{h}, \mathbf{i})$ Failure rate $(\mathbf{h})$ and latency $(\mathbf{i})$ of spikes produced by the STD-only (black bars) or STP + STD template (red bars) are compared $(n=12)$. The asterisks indicate statistical significance $(P<0.0001$ in both cases, two-tailed paired Student's $t$-tests). Error bars denote \pm s.e.m.

In the squid axon, Cole and Moore ${ }^{27}$ first described that hyperpolarization of membrane potential slowed down activation of $I_{\mathrm{k}}$. It initiated the debate whether this so-called 'Cole-Moore effect' lay in the channel gating itself or could be resulted from more than one types of $\mathrm{K}^{+}$channels with distinct activation kinetics and voltage dependence in the squid axon ${ }^{39-41}$. Subsequent studies on the voltage dependence of gating currents (that is, generated by the charge transfer of voltage sensor) in cut-open oocytes expressing homomeric Shaker channels demonstrated that the 'Cole-Moore effect' could be accounted for by the gating itself ${ }^{25,26}$. Using both native Kvs in nerve terminals and homogeneous recombinant Kvs in cell lines, we have provided concrete evidence that preceding depolarization can accelerate activation of Kvs as a result of a left shift in the $I-V$ curve and lead to a facilitation of $I_{\mathrm{k}}$ (Figs 1-4). By applying the Markov modelling of $\mathrm{Kv}$ kinetics including six gating states (Fig. 5), we have further revealed that the key determinant of the 'Cole-Moore effect' is the deactivation time constant $(\beta)$ of Kvs, 
which likely gives rise to heterogeneity in the magnitude and time course of facilitation. We interpret our observations as such that preceding activity shifts a relative fraction of Kvs to different intermediate closed states of the same channels. Compared with the resting closed state $\left(\mathrm{C}_{0}\right)$, the intermediate closed states $\left(\mathrm{C}_{1}-\right.$ $\mathrm{C}_{4}$ ) have lower energy barrier to reach the open state $(\mathrm{O})$ and hence display a lower activation threshold and faster activation kinetics. Although the 'Cole-Moore effect' has been intensively debated as an interesting biophysical phenomenon since 1960s, its physiological relevance remains unknown. Our study brings to light the significant roles of the 'Cole-Moore effect', more specifically, the intermediate closed states of Kvs, in determining presynaptic AP waveform and short-term synaptic plasticity. We suggest that the classical 'Cole-Moore effect' of Kvs can fully account for $I_{\mathrm{k}}$ facilitation in repetitive firing at the calyx of Held nerve terminal and perhaps other fast-spiking neurons ${ }^{42,43}$.

Instead of standard square voltage steps, we have used AP-like ramps or real APs to explore the gating behaviours of Kvs and their activity-dependent facilitation. In a few studies, where AP waveforms had been applied to cortical neurons, Kvs appeared to undergo use-dependent inactivation during high-frequency trains, leading to frequency-dependent spike broadening $35,44,45$. Such a discrepancy may be because of the differences in the AP waveform as well as composition of Kvs associated with different neuronal populations, as we have demonstrated that $\mathrm{KvF}$ is strongly dependent on the AP amplitude, voltage and subtype of Kvs (Figs 1 and 2). Fast-spiking neurons characterized in this study usually contain delayed rectifier Kvs such as Kv3.1 and Kv1.1/1.2 that show little inactivation. High density of these fastgating delayed rectifiers instates brief APs and minimizes usedependent reduction in the spike amplitude during highfrequency firing to promote the manifestation of $I_{\mathrm{K}}$ facilitation. Conversely, in slow-spiking neurons, such as hippocampal CA1 neurons, rapid inactivating Kvs (for example, A-type currents or C-type inactivation) may dominate and cause severe usedependent adaptation of spikes, during which the functional expression of $\mathrm{KvF}$ can be masked, albeit the fact that these channels do facilitate in response to closely adjacent APs (Supplementary Fig. 2).

It is worth noting that use-dependent decline in the spike amplitude during repetitive activity has traditionally been attributed to rapid inactivation of $\mathrm{Na}^{+}$channels. Surprisingly, under our simulation environment, frequency-dependent facilitation of Kvs actively contributes to the attenuation of the AP amplitude and DAP by counteracting $I_{\mathrm{Na}}$-induced depolarization (Fig. 6). Hence, KvF may become another factor to be considered for interpreting complex AP firing patterns at high rates in addition to classical inactivation of $\mathrm{Na}^{+}$channels. By paired recordings from pre- and postsynaptic compartments of the calyx of Held synapse, we further demonstrate that the subtle decrease in the spike amplitude and inter-spike plateau potential because of facilitation of $I_{\mathrm{K}-\mathrm{LT}}$ strongly impacts $I_{\mathrm{Ca}}$ and residual $\mathrm{Ca}^{2+}$ buildup in the nerve terminal and ultimately quantal output during high-frequency train stimulation (Fig. 7). It is also possible that heterogeneous levels of K-LT in morphologically distinct calyces can modulate presynaptic APs differently, leading to divergent forms of short-term facilitation and depression phenotypes as previously described ${ }^{46}$. Taking together, this study adds a new variable to the mechanistic underpinnings of short-term synaptic plasticity, aside from several other proposed mechanisms ${ }^{17,47}$.

In summary, our experimental evidence and computer simulations have led us to conclude that neural activities prime presynaptic Kvs into the intermediate closed states before subsequent stimuli arrive, likely owing to different positions of four voltage sensors (S4) within each Kvs ${ }^{13,48}$. These sensors not only gate Kvs but also serve as direct decoders for the frequency of afferent axonal activity, which drive facilitation of $I_{\mathrm{K}}$ to dampen depolarization and consequentially influence $\mathrm{Ca}^{2+}$ dependent short-term plasticity and postsynaptic spiking fidelity. The multifaceted function of voltage sensors may be applicable to other voltage-gated ion channels as well. Such an intrinsic mechanism of Kvs is of essential importance for fine-tuning spike waveform, inter-spike potential and synaptic input-output relationship during high-frequency neurotransmission at the calyx of Held and other fast central synapses.

\section{Methods}

Slice preparation. Mice were housed in the facility certified by the Canadian Council of Animal Care and were used for this study according to a protocol approved by the Hospital for Sick Children Animal Care Committee. Brainstem slices were prepared from postnatal (P) 16 to 19-day-old animals, as previously described $^{49}$. After decapitation with a small guillotine, brains were immediately immersed into semi-frozen artificial cerebral spinal fluid (ACSF) containing (in $\mathrm{mM}$ ): $\mathrm{NaCl}$ (125), $\mathrm{KCl}$ (2.5), glucose (10), $\mathrm{NaH}_{2} \mathrm{PO}_{4}$ (1.25), Na-pyruvate (2), myoinositol (3), ascorbic acid (0.5), $\mathrm{NaHCO}_{3}(26), \mathrm{MgCl}_{2}$ (1) and $\mathrm{CaCl}_{2}$ (2) at a pH of 7.3 when oxygenated $\left(95 \% \mathrm{O}_{2}\right.$ and $\left.5 \% \mathrm{CO}_{2}\right)$ followed by rapid dissection.

Hippocampal and cerebella slices were taken from 6 to 8 -week-old mice in ice-cold solution including (in $\mathrm{mM}$ ): $\mathrm{NaCl}$ (81.2), $\mathrm{NaHCO}_{3}$ (23.4), sucrose (69.9), glucose (23.3), $\mathrm{KCl}$ (2.4), $\mathrm{NaH}_{2} \mathrm{PO}_{4}(1.4), \mathrm{MgCl}_{2}(6.7)$ and $\mathrm{CaCl}_{2}(0.5)$ at a $\mathrm{pH}$ of 7.3. Transverse slices of the auditory brainstem containing the medial nucleus of the trapezoid body or parasagittal slices including cerebellum and hippocampus were cut at a thickness of $200-250 \mu \mathrm{m}$ using a microtome (VT1000S, Leica) and incubated in ACSF at $37^{\circ} \mathrm{C}$ for one hour before experimentation.

CHO cells expressing Kv1.1\&1.2 or Kv3.1 channels. CHO cells (ATCC CCL-61) were maintained in $60 \mathrm{~mm}$ tissue culture dishes (Nunc, Thermo Fisher Scientific) containing $5 \mathrm{ml}$ of Ham's F12 media (Wisent) supplemented with $10 \%$ fetal bovine serum and $1 \%$ penicillin-streptomycin (Wisent); and grown at $37^{\circ} \mathrm{C}$ and $5 \% \mathrm{CO}_{2}$. Cells were split twice weekly to prevent overgrowth. Cells were split by removing the culture medium, washing the cells once with $10 \mathrm{ml}$ phosphate-buffered saline (Wisent) and then incubating with $1 \mathrm{ml}$ trypsin-EDTA $(0.25 \%)$ for $3 \mathrm{~min}$ at room temperature. Subsequently, $5 \mathrm{ml}$ of culture medium was added to the dish, and cells were dissociated mechanically. Then $0.5 \mathrm{ml}$ of medium containing dissociated cells $\left(\sim 2 \times 10^{5}\right.$ cells) was added to a new dish. CHO cells $\left(1 \times 10^{4}\right.$ cells per $\left.\mathrm{cm}^{2}\right)$ were plated 1 day before transfection onto coverslips coated with poly-d-lysine $\left(50 \mu \mathrm{g} \mathrm{ml}^{-1}\right.$, Sigma). Transfection was carried out using the FuGene HD (Roche) method with Kv1.1-RFP:Kv1.2-GFP ratio of 1:1 (gift from Drs Gang Xie and John Roder, University of Toronto). After transfection, cells were maintained in Ham's F12 media for $48 \mathrm{~h}$ before experiments. CHO cells with stable expression of Kv3.1 channels were generously provided by Dr Leonard K Kaczmarek (Yale University).

Electrophysiology. For recordings from brain slices, ACSF was supplemented with bicuculline $(10 \mu \mathrm{M})$ and strychnine $(1 \mu \mathrm{M})$ to block inhibitory inputs. To isolate $\mathrm{K}^{+}$currents, TTX $(0.5 \mu \mathrm{M})$ and $\mathrm{CdCl}_{2}(20 \mu \mathrm{M})$ were added to block Na and $\mathrm{Ca}^{2}+$ channels. Intracellular solution for recording $\mathrm{K}^{+}$currents and APs contained the following (in $\mathrm{mM}$ ): K-gluconate (97.5), $\mathrm{KCl}(32.5)$, EGTA (0.5) or BAPTA (30), HEPES (40) and $\mathrm{MgCl}_{2}$ (1), $\mathrm{pH}$ 7.3. To isolate presynaptic $\mathrm{Ca}^{2}+$ currents, TTX $(0.5 \mu \mathrm{M})$, TEA $(10 \mathrm{mM})$, and 4 -aminopyridine $(0.3 \mathrm{mM})$ were applied extracellularly to inhibit $\mathrm{Na}^{+}$and $\mathrm{K}^{+}$channels. Intracellular solution for recording presynaptic $\mathrm{Ca}^{2}+$ currents included (in $\mathrm{mM}$ ): $\mathrm{CsCl}$ (110), HEPES (40), EGTA (0.5), $\mathrm{MgCl}_{2}$ (1), ATP (2), GTP (0.5), phosphocreatine (12), TEA (20) and K-glutamate (3), $\mathrm{pH}$ adjusted to 7.3 with $\mathrm{CsOH}$. For postsynaptic recordings, intracellular solution contained (in mM): K-gluconate (97.5), CsCl (32.5), EGTA (5), HEPES (10), $\mathrm{MgCl}_{2}$ (1), TEA (30) and lidocaine $N$-ethyl bromide (3), pH 7.3. Patch electrodes typically had resistances of 4-6 and 2.5-3 M $\Omega$ for pre- and postsynaptic recordings, respectively. For whole-cell voltage-clamp recordings, preand postsynaptic series resistances were $6-15 \mathrm{M} \Omega(<10 \mathrm{M} \Omega$ in most cases $)$ and 3-6 M $\Omega$, respectively, and compensated to $90 \%$. The respective holding potential for pre- or postsynaptic neurons was -80 and $-60 \mathrm{mV}$. Presynaptic $\mathrm{K}^{+}$and $\mathrm{Ca}^{2+}$ currents were evoked by the voltage-command protocols indicated in the text, and leak subtraction was done with an online $\mathrm{P} / 4$ procedure. To validate the online leak $\mathrm{P} / \mathrm{N}$ subtraction, we recorded raw currents evoked by a typical AP train at $400 \mathrm{~Hz}$ without $\mathrm{P} / \mathrm{N}$ subtraction after blocking $\mathrm{Na}^{+}$channels with TTX and $\mathrm{Ca}^{2}+$ channels with $\mathrm{CdCl}_{2}$. We subtracted these currents with those obtained with additional blockers for K-HT (TEA) and K-LT (DTX). By comparing the subtracted TEA and DTX-sensitive currents with $\mathrm{K}^{+}$currents recorded with online $\mathrm{P} / \mathrm{N}$ correction, we found that they were almost identical in the amplitude and more importantly in the profile of facilitation over the train stimuli (Supplementary Fig. 4). For experiments where real APs were used as voltage command (Fig. 3 and Supplementary Figs 3 and 4), we first recorded APs from a P17 calyx at a sampling rate of $50 \mathrm{kHz}$ in current-clamp configuration by stimulating afferent axon fibres using a bipolar platinum electrode delivered through a stimulator (Master-8, 
A.M.P. Instruments). After manually removing stimulation artefacts preceding the APs, the digitized values were fed back into the amplifier as voltage-command templates (Axon Text File) through pClamp 9 software (Molecular Devices) at the same sampling frequency as their acquisition. Similarly, the current-command templates in Fig. 8 were generated by reversing the polarity of previously obtained EPSCs in response to $400 \mathrm{~Hz}$ train stimuli with normal or attenuated K-LT facilitation (Fig. 7) and converting these currents to conductance by $G=I /\left(V_{\mathrm{m}}-E_{\text {rev }}\right)$, where $V_{\mathrm{m}}$ and $E_{\text {rev }}$ were set at -70 and $0 \mathrm{mV}$, respectively, for postsynaptic neurons. The digitized conductance templates (Text File) were injected into these neurons to evoke APs with an amplifier (AXOPATCH 200B, Molecular Devices) and a digitizer (CED 1401, Cambridge Electronic Design).

$\mathrm{CHO}$ cells transfected with Kv1.1\&1.2 were positively identified with red/green fluorescence illuminated briefly by a mercury burner (Olympus). Recordings from CHO cells expressing Kv3.1 or Kv1.1\&1.2 (Fig. 4 and Supplementary Fig. 5) were made in the extracellular solution containing (in $\mathrm{mM}$ ): $\mathrm{NaCl}(140), \mathrm{KCl}(2.5)$, $\mathrm{CaCl}_{2}$ (1.3), HEPES (10) and glucose (33), $\mathrm{pH}$ 7.3. And the intracellular solution was the same as used for recording $\mathrm{K}^{+}$currents from brain slices. The holding potential was set at $-90 \mathrm{mV}$ and series resistance was 3-6 M , compensated to $90 \%$.

Most of the electrophysiological experiments were performed at room temperature $\left(\sim 22^{\circ} \mathrm{C}\right)$, except for several sets of recordings of $\mathrm{K}^{+}$currents from $\mathrm{CHO}$ cells or cerebellar neurons obtained at $35^{\circ} \mathrm{C}$ using an inline heater with a feedback thermistor (TC-324B, Warner Instruments). All the recordings (except for those in Fig. 8) were acquired online, filtered at $4 \mathrm{kHz}$, digitized at 50 or $100 \mathrm{kHz}$ with a dual-channel amplifier (MultiClamp 700A, Molecular Devices) and digitizer (Digidata 1322A, Molecular Devices). Reagents were obtained from Sigma (St Louis, MO), Tocris Cookson (Bristol, UK) and Alomone Labs (Jerusalem, Israel).

Data analysis. Data were analysed offline with pClamp 9 (Molecular Devices), MiniAnalysis (Synaptosoft) and Excel XP (Microsoft). PPF was calculated by dividing the amplitude or area integral of the second $\mathrm{K}^{+}$current by that of the first one during a paired-pulse stimulation. The decay time course of PPF over increasing paired-pulse intervals was described with a single exponential function: $\mathrm{f}(t)=\sum A^{*} \exp (-t / \tau)+C$. Time constants $(\tau)$ were given in respective figures. In Fig. 7, the amplitude of $I_{\mathrm{Ca}}$ and $I_{\mathrm{EPSC}}$ elicited by each AP train was normalized to that of the first currents. The time course of normalized $I_{\mathrm{Ca}}$ and $I_{\mathrm{EPSC}}$ over an AP train was fitted with a single or double exponential function. Statistical tests of significance were two-tailed, paired Student's $t$-tests with a $P$ value cutoff of $<0.05$. Data were expressed as the mean \pm s.e.m from a population of cells $(n)$.

Computer simulations. Kinetic fittings of activation and deactivation of $I_{\mathrm{K}-\mathrm{HT}}, I_{\mathrm{K}}$ $\mathrm{LT}$ and $I_{\mathrm{Na}}$ from outside-out patches in voltage-clamp experiments were performed using the Markov model in combination with particle swarm optimization and golden section search-based algorithms to determine the optimal match to macroscopic currents and to extract all free-rate parameters (Table 1 and

Supplementary Fig. 6). Details of computational methods were previously described $^{16,28}$. All conductances were incorporated into the modified $\mathrm{H}-\mathrm{H}$ model cell for simulating APs at different frequencies. The differential equations for the kinetic modelling were solved numerically, using a fifth-order Runge-Kutta integration method. The integrating routines were written and executed with software CeL (Huazhong University of Science and Technology, Wuhan, China) and compiled with the $\mathrm{C}++$ compiler to run under Windows XP (www.HustCeL.com).

\section{References}

1. Hodgkin, A. L. A. \& Huxley, A. F. A. Currents carried by sodium and potassium ions through the membrane of the giant axon of Loligo. J. Physiol. 116, 449-472 (1952).

2. Armstrong, C. M. \& Bezanilla, F. Currents related to movement of the gating particles of the sodium channels. Nature 242, 459-461 (1973).

3. Aldrich, R. W., Corey, D. P. \& Stevens, C. F. A reinterpretation of mammalian sodium channel gating based on single channel recording. Nature 306, 436-441 (1983).

4. Llinas, R. R. The intrinsic electrophysiological properties of mammalian neurons: insights into central nervous system function. Science 242, 1654-1664 (1988).

5. Serrano, J. R., Perez-Reyes, E. \& Jones, S. W. State-dependent inactivation of the alpha1G T-type calcium channel. J. Gen. Physiol. 114, 185-201 (1999).

6. Raman, I. M., Gustafson, A. E. \& Padgett, D. Ionic currents and spontaneous firing in neurons isolated from the cerebellar nuclei. J. Neurosci. 20, 9004-9016 (2000).

7. Hille, B. in Ion Channels of Excitable Membranes 3rd edn, 814 (University of Washington, 2001).

8. Beck, E. J., Bowlby, M., An, W. F., Rhodes, K. J. \& Covarrubias, M. Remodelling inactivation gating of Kv4 channels by KChIP1, a small-molecular-weight calcium-binding protein. J. Physiol. 538, 691-706 (2002).
9. Maurice, N. et al. D2 dopamine receptor-mediated modulation of voltagedependent $\mathrm{Na}+$ channels reduces autonomous activity in striatal cholinergic interneurons. J. Neurosci. 24, 10289-10301 (2004).

10. Magistretti, J. \& Alonso, A. Multiple conductance substates in pharmacologically untreated $\mathrm{Na}(+)$ channels generating persistent openings in rat entorhinal cortex neurons. J. Membr. Biol. 214, 165-180 (2006).

11. Bean, B. P. The action potential in mammalian central neurons. Nat. Rev. Neurosci. 8, 451-465 (2007).

12. Coetzee, W. A. et al. Molecular diversity of $\mathrm{K}+$ channels. Ann. N. Y. Acad. Sci. 868, 233-285 (1999).

13. Tao, X., Lee, A., Limapichat, W., Dougherty, D. A. \& MacKinnon, R. A gating charge transfer center in voltage sensors. Science 328, 67-73 (2010).

14. Gurkiewicz, M. \& Korngreen, A. A numerical approach to ion channel modelling using whole-cell voltage-clamp recordings and a genetic algorithm. PLoS Comput. Biol. 3, e169 (2007).

15. Milescu, L. S., Akk, G. \& Sachs, F. Maximum likelihood estimation of ion channel kinetics from macroscopic currents. Biophys. J. 88, 2494-2515 (2005).

16. Wang, W. et al. Optimal estimation of ion-channel kinetics from macroscopic currents. PLoS ONE 7, e35208 (2012).

17. von, G. H. \& Borst, J. G. Short-term plasticity at the calyx of held. Nat. Rev. Neurosci. 3, 53-64 (2002).

18. Wang, L. Y., Gan, L., Forsythe, I. D. \& Kaczmarek, L. K. Contribution of the $\mathrm{Kv} 3.1$ potassium channel to high-frequency firing in mouse auditory neurones. J. Physiol. 509(Pt 1): 183-194 (1998).

19. Wang, L. Y. \& Kaczmarek, L. K. High-frequency firing helps replenish the readily releasable pool of synaptic vesicles. Nature 394, 384-388 (1998).

20. Trussell, L. O. Modulation of transmitter release at giant synapses of the auditory system. Curr. Opin. Neurobiol. 12, 400-404 (2002).

21. Brew, H. M. \& Forsythe, I. D. Two voltage-dependent $\mathrm{K}+$ conductances with complementary functions in postsynaptic integration at a central auditory synapse. J. Neurosci. 15, 8011-8022 (1995).

22. Ishikawa, T. et al. Distinct roles of Kv1 and Kv3 potassium channels at the calyx of Held presynaptic terminal. J. Neurosci. 23, 10445-10453 (2003).

23. Dodson, P. D. \& Forsythe, I. D. Presynaptic K + channels: electrifying regulators of synaptic terminal excitability. Trends Neurosci. 27, 210-217 (2004).

24. Kim, J. H., Kushmerick, C. \& von, G. H. Presynaptic resurgent Na + currents sculpt the action potential waveform and increase firing reliability at a CNS nerve terminal. J. Neurosci. 30, 15479-15490 (2010).

25. Perozo, E., Papazian, D. M., Stefani, E. \& Bezanilla, F. Gating currents in Shaker $\mathrm{K}+$ channels. Implications for activation and inactivation models. Biophys. J. 62, 160-168 (1992).

26. Bezanilla, F., Perozo, E. \& Stefani, E. Gating of Shaker K + channels: II. The components of gating currents and a model of channel activation. Biophys. J. 66, 1011-1021 (1994).

27. Cole, K. S. \& Moore, J. W. Potassium ion current in the squid giant axon: dynamic characteristic. Biophys. J. 1, 1-14 (1960).

28. Sun, L. et al. Differential regulation of action potentials by inactivating and noninactivating BK channels in rat adrenal chromaffin cells. Biophys. J. 97, 1832-1842 (2009).

29. Dodge, Jr F. A. \& Rahamimoff, R. Co-operative action a calcium ions in transmitter release at the neuromuscular junction. J. Physiol. 193, 419-432 (1967).

30. Fedchyshyn, M. J. \& Wang, L. Y. Developmental transformation of the release modality at the calyx of Held synapse. J. Neurosci. 25, 4131-4140 (2005).

31. Yang, Y. M. \& Wang, L. Y. Amplitude and kinetics of action potential-evoked $\mathrm{Ca} 2+$ current and its efficacy in triggering transmitter release at the developing calyx of held synapse. J. Neurosci. 26, 5698-5708 (2006).

32. Yang, Y. M. et al. GluA4 is indispensable for driving fast neurotransmission across a high-fidelity central synapse. J. Physiol. 589, 4209-4227 (2011).

33. Dobrunz, L. E. \& Stevens, C. F. Heterogeneity of release probability, facilitation, and depletion at central synapses. Neuron 18, 995-1008 (1997).

34. Awatramani, G. B., Price, G. D. \& Trussell, L. O. Modulation of transmitter release by presynaptic resting potential and background calcium levels. Neuron 48, 109-121 (2005).

35. Geiger, J. R. \& Jonas, P. Dynamic control of presynaptic $\mathrm{Ca}(2+)$ inflow by fast-inactivating $\mathrm{K}(+)$ channels in hippocampal mossy fiber boutons. Neuron 28, 927-939 (2000).

36. Xu, J., He, L. \& Wu, L. G. Role of $\mathrm{Ca}(2+)$ channels in short-term synaptic plasticity. Curr. Opin. Neurobiol. 17, 352-359 (2007).

37. Chaudhuri, D., Issa, J. B. \& Yue, D. T. Elementary mechanisms producing facilitation of Cav2.1 (P/Q-type) channels. J. Gen. Physiol. 129, 385-401 (2007)

38. Lee, A. et al. $\mathrm{Ca} 2+/$ calmodulin binds to and modulates $\mathrm{P} / \mathrm{Q}$-type calcium channels. Nature 399, 155-159 (1999).

39. Bezanilla, F., Perozo, E., Papazian, D. M. \& Stefani, E. Molecular basis of gating charge immobilization in Shaker potassium channels. Science 254, 679-683 (1991).

40. Taglialatela, M. \& Stefani, E. Gating currents of the cloned delayed-rectifier K + channel DRK1. Proc. Natl Acad. Sci USA 90, 4758-4762 (1993). 
41. Clay, J. R. A simple model of $\mathrm{K}+$ channel activation in nerve membrane. J. Theor. Biol. 175, 257-262 (1995).

42. Klug, A. \& Trussell, L. O. Activation and deactivation of voltage-dependent $\mathrm{K}+$ channels during synaptically driven action potentials in the MNTB. J. Neurophysiol. 96, 1547-1555 (2006).

43. Johnston, J. et al. Initial segment Kv2.2 channels mediate a slow delayed rectifier and maintain high frequency action potential firing in medial nucleus of the trapezoid body neurons. J. Physiol. 586, 3493-3509 (2008).

44. Raman, I. M. \& Bean, B. P. Ionic currents underlying spontaneous action potentials in isolated cerebellar Purkinje neurons. J. Neurosci. 19, 1663-1674 (1999).

45. Swensen, A. M. \& Bean, B. P. Ionic mechanisms of burst firing in dissociated Purkinje neurons. J. Neurosci. 23, 9650-9663 (2003)

46. Grande, G. \& Wang, L. Y. Morphological and functional continuum underlying heterogeneity in the spiking fidelity at the calyx of Held synapse in vitro. J. Neurosci. 31, 13386-13399 (2011).

47. Zucker, R. S. \& Regehr, W. G. Short-term synaptic plasticity. Annu. Rev. Physiol. 64, 355-405 (2002).

48. MacKinnon, R. Potassium channels. FEBS Lett. 555, 62-65 (2003).

49. Barnes-Davies, M. \& Forsythe, I. D. An in-vitro thin slice preparation of the rat brain-stem for patch-clamp recordings of synaptic currents from auditory neurons. J. Physiol. 467, 240 (1993).

\section{Acknowledgements}

This work was supported by individual Operating Grants from the Canadian Institutes of Health Research and Canada Research Chair (to L.-Y.W.); China-Canada Joint Health
Research Initiative Grant (to L.-Y.W. and Z.Z.); The National Basic Research Program of China (2010CB529804) and the National Science Foundation of China (30971179 and 31170814; to J.D.). We thank Drs Michael W. Salter, Steve Prescott and Milton Charlton for their critical inputs to a previous version of this manuscript, Stéphanie Ratté for her technical support with dynamic clamp, Lu Han for transfecting and maintaining $\mathrm{CHO}$ cell lines, and other members of the Wang Laboratory for their assistance and discussions.

\section{Author contributions}

Y.-M.Y. designed and executed the electrophysiological recordings; W.W., M.J.F., Z.Z., J.D. and L.-Y.W. performed computer simulations; Y.-M.Y. and L.-Y.W. wrote the manuscript; and L.-Y.W. and J.D. devised and supervised this study.

\section{Additional information}

Supplementary Information accompanies this paper at http://www.nature.com/ naturecommunications

Competing financial interests: The authors declare no competing financial interests.

Reprints and permission information is available online at http://npg.nature.com/ reprintsandpermissions/

How to cite this article: Yang, Y.-M. et al. Enhancing the fidelity of neurotransmission by activity-dependent facilitation of presynaptic potassium currents. Nat. Commun. 5:4564 doi: 10.1038/ncomms5564 (2014). 